\title{
Energy Optimization of a Laser-Powered Hovering-UAV Relay in Optical Wireless Backhaul
}

This paper was downloaded from TechRxiv (https://www.techrxiv.org).

\section{LICENSE}

CC BY 4.0

SUBMISSION DATE / POSTED DATE

$27-11-2021 / 06-12-2021$

\section{CITATION}

Bashir, Muhammad Salman; Alouini, Mohamed-Slim (2021): Energy Optimization of a Laser-Powered Hovering-UAV Relay in Optical Wireless Backhaul. TechRxiv. Preprint.

https://doi.org/10.36227/techrxiv.17087309.v1

$\mathrm{DOI}$

10.36227/techrxiv.17087309.v1 


\title{
Energy Optimization of a Laser-Powered Hovering-UAV Relay in Optical Wireless Backhaul
}

\author{
Muhammad Salman Bashir, Senior Member, IEEE and Mohamed-Slim Alouini, Fellow, IEEE
}

\begin{abstract}
Due to their flexibility and low cost deployment, unmanned aerial vehicles (UAV) will most likely act as base stations and backhaul relays in the next generation of wireless communication systems. However, these UAVs-in the untethered mode - can only operate for a finite time due to limited energy they carry in their batteries. In free-space optical communications, one solution is to transport both the data and the energy from the source to the UAV through the laser beam-a concept known as simultaneous lightwave information and power transfer (SLIPT). In this study, we have analyzed the SLIPT scheme for laser-powered decode-and-forward UAV relays in an optical wireless backhaul. The major goal of this study is to optimally allocate the received beam energy between the decoding circuit, the transmitting circuit and the rotor block of the relay in order to maximize a quality-of-service metric such as maximum achievable rate, outage or error probabilities. As expected, we note that the optimal power allocation depends heavily on the source-relay and relay-destination channel conditions. In the final part of this study, we have maximized the operational time of the UAV relay given that the maximum achievable rate stays above a certain threshold in order to meet a minimum quality-of-service requirement.
\end{abstract}

\section{Index Terms}

Decode-and-forward relay, energy harvesting, error probability, free-space optics, hovering unmanned aerial vehicle, laser-powered unmanned aerial vehicle, maximum achievable rate, optimal power allocation, outage probability, simultaneous lightwave information and power transfer

\section{INTRODUCTION}

Free-space optics — or free-space laser communications - is slated to be a promising technology for the support of higher data rates in the backhaul of next generation of wireless communication systems. One 
of the major advantages of laser communications is the availability of large bands in the optical domain of the electromagnetic spectrum that can help achieve transmission rates in tens of of Gigabits per second. Another advantage of FSO is the realization of low-cost and small-sized noncoherent energy detecting receivers that may be easily mounted on a small aerial platform such as a UAV.

It is expected that UAVs will play an important role in the future generation of wireless communications [1] just as they are set to revolutionize almost all the aspects of a modern human society: from smart package delivery and traffic monitoring to surveillance and security applications to name a few. One of the major applications of UAVs in a wireless communication architecture will be the replacement of traditional base stations-which are fixed on the ground and therefore stationary-with flying aerial vehicles that will offer much more flexibility to a system designer in terms of maximizing a certain quality-of-service metric (maximum achievable rate, bit error rate) of the communication system [2]. Such UAVs will also find applications as hovering relays in the backhaul of a future communication system due to their relative ease and quick deployment in a dense urban environment [3].

One of the major hurdles in the extensive deployment of UAVs for wireless communications is the limited amount of energy that is stored on an on-board battery. Thus, once the stored energy is exhausted, the communication link faces a disruption because the UAV has to alight on the ground charging station to recharge the battery. This problem may be resolved by two possible solutions: i) either by using a tether cable that acts both as a stabilizing force as well as a source of energy [4] to power the UAV, or, ii) by employing beam-powered scheme (commonly known as Simultaneous Wireless Information and Power Transfer or SWIPT for short) in which the radio or laser beam is used both for data transmission as well as for supplying power to the UAV battery [5]. Even though the tethered scheme provides a continuous source of power supply, it limits the region of operation or the coverage area of the UAV. The SWIPT scheme allows far more flexibility in this regard; however, if the supplied laser power is not enough, the UAV may still experience some downtime that will limit the uninterrupted operational time of the UAV.

\section{A. Motivation for Current Study}

In this paper, we have analyzed the energy optimization problem for a laser-powered hovering UAV decode-and-forward (DF) relay in a dual-hop communications link. The laser-powered scheme uses the same beam for data transmission as well as for energy supply to the UAV, and is commonly known as simultaneous lightwave information and power transfer scheme-or SLIPT for short-in literature. In the context of SLIPT and DF relays, an important problem is the optimal allocation of the received energy 
between the following three channels in the relay with the aim to optimize a certain quality-of-service metric of the system: i) energy used for symbol decoding at the relay, ii) energy required for symbol transmission to the destination or the next relay, and iii) the energy needed to power the rotors. For instance, if one allocates too much energy for symbol decoding at the relay, then a small fraction of the remaining energy is allocated for symbol transmission to the destination or for rotor power. This will likely deteriorate the error rate or the data rate performance at the destination and will also reduce the uptime of the UAV. Hence, an optimal allocation of energy between all three channels at the relay is needed in order to maximize the overall system performance.

\section{Background Literature Review and Contributions of Current Study}

Sixth generation and beyond communication networks envisage a world where the number of mobile communication devices will far exceed the number of fixed or stationary terminals. Therefore, energy harvesting for mobile devices constitutes one of the major areas of research on future networks, and in this regard, the ideas behind SWIPT and SLIPT have garnered tremendous interest in the last few years.

An important, high-level, paper on SWIPT is [5] wherein the authors lay out a concise introduction and discuss preliminary ideas on the technology behind SWIPT. An important article on SLIPT—which is actually the basis of study in this paper-is [6] in which the authors discuss the optimization problems concerning the SLIPT scheme for airborne radio access networks. In their work, the energy allocation for a UAV-based access network is optimized in order to maximize the individual achievable rates and the sum rate of spectrum users. The same authors in [7] apply SLIPT in order to maximize system performance for indoor internet-of-things applications. They solve a fundamental optimization problem in which the harvested energy is maximized under the constraint that the Quality of Service does not go below a certain threshold. Specifically in the domain of indoor visible light communications (VLC), the study [8] focuses on receiver design in the domains of time and signal components and photoelectric converters. The same study also applies SLIPT to various network topologies and communication technologies such as multiple-input multiple-out and hybrid radio-VLC frequency communications. Another paper that applies SLIPT to visible light communications is [9] in which the authors investigate the downlink unicast signal transmission of multi-LED multi-user SLIPT networks. In their paper, they study the total transmit power minimization problem under the the constraints of achievable rate requirement, minimum energy harvesting and dimming control requirements. Finally, another study that applies SLIPT to VLC systems is [10] in 
which the authors optimize the energy harvesting and data rate performance of multiple users under lighting constraints.

Now we discuss some references concerned with SWIPT based energy harvesting relays. In [11], the authors have obtained optimal precoders at the source and the relay to maximize the achievable rate of the end-to-end link of a dual hop relay scheme. Additionally, they have also investigated the effect of source transmit power and the position of the relay in the link on the maximum achievable rate. In [12], the authors discuss the SWIPT mechanism for a multihop decode-and-forward relaying scheme in which they minimize the transmit power under the constraints of some minimum quality-of-service threshold. Additionally, they maximize the achievable rate of the system by optimally allocating power at each relay in the multihop link. The authors in [13] consider SWIPT for a two-way relay network where the minimize the outage probability by jointly optimizing power allocation of the source nodes, power splitting at relay nodes and the time allocation of each duration. The authors in [14] consider laser-powered UAVs for stochastic geometry-based analysis of airborne base stations. Finally, the study in [15] considers optimal placement of UAV relays in a multihop relay scheme so that the end-to-end error and outage probabilities in an optical wireless backhaul can be minimized.

For a general discussion on multihop amplify-and-forward and decode-and-forward relays, the interested reader is referred to [16], [17]. For a discussion on pointing error, acquisition and beam tracking in FSO, the reader is referred to $[18]-[25]$.

\section{A. Contributions of This Paper}

In this manuscript, we have analyzed and optimized the performance of a dual hop relay scheme in an optical wireless communication system where the relay is not powered by any external source, and a SLIPTbased mechanism is adopted in order to power the UAV. In this regard, we have solved the optimization problems that involve the optimal allocation of received power between the following channels in the relay: i) the decoding channel, ii) the transmitting channel, and iii) the channel supplying power to the rotors. The optical channel that we have considered is mainly affected by the pointing error due to hover of the UAV relay [3], and the performance metrics that we have optimized are the maximum achievable rate or ergodic capacity, the outage probability and the error probability. In the final part of the paper, we have also attempted to maximize the operational or uptime of the UAV under maximum achievable rate constraint. 
In order to reduce complexity of optimization, we have derived closed-form expressions for the ergodic capacity and outage probability of the system in this manuscript. Finally, under the assumption that the beamwidth is much larger than the magnitude of pointing error for the relay-destination link, the optimization problems become much more tractable and can be solved analytically.

\section{B. Organization of This Paper}

This paper is organized as follows. Section III lays out the channel model for the dual-hop relay scheme in detail along with all the assumptions. Section [II-C describes the architecture of the energy harvesting relay that works on the principle of SLIPT. Section IV derives the maximum achievable rate, outage probability and error probability expressions for the SLIPT relay based dual-hop channel, and Section $\mathrm{V}$ deals with the optimal energy allocation problem of the SLIPT relay. Section VI explains the simulation or experimental results, and Section VII concludes this study with important points and future directions.

\section{Channel Model}

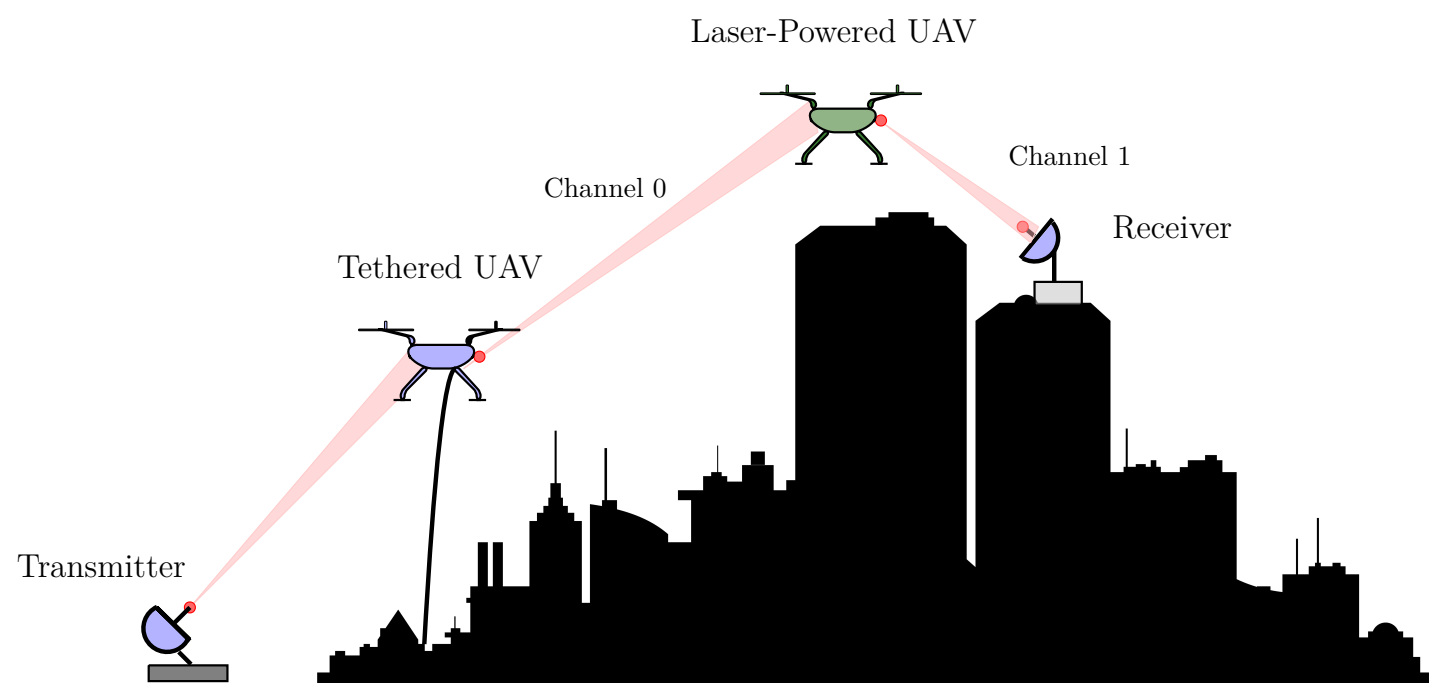

Fig. 1. This figure shows a laser-powered UAV relay that connects the source (tethered UAV) with the destination receiver in a dense urban environment.

We consider a channel characterised by dense urban environment as shown in Fig. 1 where the lineof-sight between the transmitter and the destination receiver may be disrupted due to the presence of tall buildings that may be high enough to shadow the receiver completely. In order to overcome this issue, UAV based relays may be deployed that can be placed high enough so that a line-of-sight can be established. In this study, we assume a dual hop relay scheme where the source is a tethered UAV and the 
destination is a fixed receiver. For the sake of clarity, we denote the source-relay channel as Channel 0 or S-R channel, and the relay-destination channel as Channel 1 or R-D channel. We assume that the relay between the source and the destination is the energy harvesting type that derives all its power from the beam transmitted from the source (which is the tethered UAV). The source-relay-destination channel is characterised by the following set of assumptions:

1) We assume that the link distances (source-relay and relay-destination links) and the beam waists at the source and relay are small enough so that the scintillation effects due to turbulence are ignored. This argument stems from the assumption that the beam footprint for a relatively shorter link distance and a highly focused beam is smaller than the size of turbulent eddies in the atmosphere. For this scenario, the scintillation of the beam can be ignored, and the only fading the signal experiences is due to the pointing error which is caused either by beam wander or due to the angular vibrations of the hovering drone. The total pointing error is the sum of these two (independent) pointing errors. Incidentally, one of the advantages of using this simplified model is the tractability if affords in reaching closed-form expressions that simplifies the optimization complexity significantly. Had we used the traditional composite model $h=h_{a} h_{p}$ where $h_{a}$ is the channel coefficient due to scintillation, and $h_{p}$ is the channel weight due to pointing error, the resulting capacity and outage probability expressions would have been highly complicated [26]. In order to avoid complicated mathematical expressions, the authors in [26] have approximated the composite model with a much simpler expression in the large SNR regime (Equation 20 in [26]) which is quite similar to our model (equations (8) and (10)).

2) The magnitude of pointing error is assumed to follow a Rayleigh distribution. This assumption is the basis of many studies concerning the pointing error (one such study is [27]), and is attractive due to the mathematical tractability afforded by the Rayleigh distribution.

3) We have considered the beam with a Gaussian intensity profile in our analysis. At the relay receiver as well as the destination receiver, the beamwidth angle (denoted by $\theta$ ) is assumed to be greater than the angular pointing error standard deviation (denoted by $\sigma$ ). If this condition were not true, the outage probability at the receivers will become significant. Additionally, a related assumption is that the beamwidth $\theta$ is large enough so that the receiver aperture area is much smaller than the footprint of the beam at the receiver location (point receiver assumption). This assumption helps us simplify the expressions significantly in order to reach closed-form expressions. 
4) We have considered thermal noise-characterised by a Gaussian distribution—as the dominant source of noise at the receivers. The assumption is that the received signal level at the receivers is large enough so that the Gaussian approximation of a Poisson process holds, but not large enough that the signal dependent shot noise dominates the thermal noise.

5) We have assumed that highly stable UAVs are available whose angular pointing error standard deviation during hovering is close to $1 \mathrm{mrad}[3]$.

\section{A. Pointing Error Model}

Assuming a point receiver, the (random) channel coefficient between source and relay is

$$
\mathbb{h}_{0}=\frac{1}{2 w_{0}^{2}(z)} \exp \left(-\frac{R_{0}^{2}}{2 w_{0}^{2}(z)}\right) \exp (-\psi z) a_{r}^{2} \approx \frac{1}{2 \theta_{0}^{2} z^{2}} \exp \left(-\frac{R_{0}^{2}}{2 \theta_{0}^{2} z^{2}}\right) \exp (-\psi z) a_{r}^{2}
$$

where $w_{0}(z)$ is the radius of the beam at the relay which is situated at a distance $z$ from the source. The quantity $\theta_{0}$ is the angular beamwidth of the beam from the source (Channel 0 ) and for small $\theta_{0}$, it holds that $w_{0} \approx \theta_{0} z$. The factor $a_{r}$ is the radius of the relay receiver lens and we assume that $w_{0} \gg a_{r}$ (point receiver assumption). The factor $\psi$ is a constant that represents the attenuation of the signal in the medium due to scattering and absorption. The quantity $R_{0}$ is a Rayleigh random variable that describes the pointing error in Channel 0 , and its scale parameter is denoted by $\sigma_{R_{0}}$. The distribution is described by

$$
f_{R_{0}}(r)=\frac{r}{\sigma_{R_{0}}^{2}} \exp \left(-\frac{r^{2}}{2 \sigma_{R_{0}}^{2}}\right) \cdot \mathbb{1}_{[0, \infty)}(r)
$$

where $\mathbb{1}_{A}$ is the indicator function over a measurable set $A$.

It can be shown that the mean channel coefficient between source and relay is [15]

$$
\mathrm{E}\left[\mathrm{h}_{0}\right]=\frac{a_{r}^{2}}{2 z^{2}\left(\theta_{0}^{2}+\sigma_{0}^{2}\right)} \exp (-\alpha z)
$$

In a similar fashion, we define the (random) channel coefficient between relay and destination, $\mathbb{h}_{1}$, as

$$
\mathbb{h}_{1} \approx \frac{1}{2 \theta_{1}^{2}(D-z)^{2}} \exp \left(-\frac{R_{1}^{2}}{2 \theta_{1}^{2}(D-z)^{2}}\right) \exp (-\psi(D-z)) a_{d}^{2}
$$

where $D$ is the sum of S-R and R-D link lengths, $w_{1}(z) \approx \theta_{1}(D-z)$ is the beam radius at the destination, $\theta_{1}$ is the beamwidth in Channel $1, R_{1}$ is a Rayleigh random variable (with parameter $\sigma_{R_{1}}$ ) that captures the pointing error of Channel 1 , and $a_{d}$ is the radius of the destination receiver aperture. 


\section{B. Distribution of $\mathrm{h}_{1}$ and $\mathrm{h}_{0}$}

In this section, we derive the statistics of channel coefficients $h_{1}$ and $\mathbb{h}_{0}$. Let us focus first on Channel 1 (which is characterised by $h_{1}$ ). The statistics of $h_{0}$ will follow in a straightforward fashion from the result for $\mathrm{h}_{1}$.

Let $\phi_{1}$ be the angular deviation of the beam that leads to pointing error $R_{1}$ at the receiver. The quantity $R_{1} \approx \phi_{1}(D-z)$ is a Rayleigh random variable with a scale parameter (or spread factor) $\sigma_{R_{1}}$. The quantity $\sigma_{R_{1}}^{2}=\sigma_{X_{1}}^{2}=\sigma_{Y_{1}}^{2}$ where $X_{1}$ and $Y_{1}$ are zero-mean i.i.d. Gaussian random variables with a common variance which is equal to either $\sigma_{X_{1}}^{2}$ or $\sigma_{Y_{1}}^{2}$, and the relationship between $R_{1}$ and $X_{1}, Y_{1}$ is $R_{1}=\sqrt{X_{1}^{2}+Y_{1}^{2}}$. Let $\phi_{X_{1}}$ and $\phi_{Y_{1}}$ be the independent (nonsolid) angles in the $x$ and $y$ dimensions, respectively. We assume that $\phi_{X_{1}}$ and $\phi_{Y_{1}}$ are both $\mathcal{N}\left(0, \sigma_{X_{1}}^{2}\right)$. Then for a distance $D-z$ between the relay and the destination, we have that $\sigma_{X_{1}}^{2}=\sigma_{Y_{1}}^{2} \approx \sigma_{1}^{2}(D-z)^{2}$, where $\sigma_{1}$ is the standard deviation of the angular pointing error in Channel 1. This implies that $\sigma_{R_{1}}^{2}=\sigma_{1}^{2}(D-z)^{2}$.

It can be shown that the distribution of $a R_{1}{ }^{2}$ - where $a$ is a positive constant-is exponential with mean $\mathrm{E}\left[a R_{1}^{2}\right]=2 a \sigma_{R_{1}}^{2}=2 a \sigma_{1}^{2}(D-z)^{2}$. This implies that $\frac{R_{1}^{2}}{2 w_{1}^{2}(D-z)}=\frac{R_{1}^{2}}{2 \theta_{1}^{2}(D-z)^{2}}$ is exponential with mean $\frac{\sigma_{1}^{2}}{\theta_{1}^{2}}$ which is independent of $D$ and $z$.

The random variable $Y:=\exp \left(-\frac{R_{1}^{2}}{2 \theta_{1}^{2}(D-z)^{2}}\right)$ takes on values between 0 and 1 and is distributed with a CDF

$$
\begin{aligned}
F_{Y}(y) & =\operatorname{Pr}(\{Y \leq y\})=\operatorname{Pr}\left(\left\{\exp \left(-\frac{R_{1}^{2}}{2 \theta_{1}^{2}(D-z)^{2}}\right) \leq y\right\}\right)=\operatorname{Pr}\left(\left\{\frac{R_{1}^{2}}{2 \theta_{1}^{2}(D-z)^{2}}>-\ln y\right\}\right) \\
& =\exp \left(\frac{\theta_{1}^{2}}{\sigma_{1}^{2}} \ln y\right)=y^{\frac{\theta_{1}^{2}}{\sigma_{1}^{2}}} .
\end{aligned}
$$

Thus, we have that $F_{Y}(y)=y^{\theta_{1}^{2} / \sigma^{2}} \cdot \mathbb{1}_{(0,1]}(y)$ or

$$
f_{Y}(y)=\frac{\theta_{1}^{2}}{\sigma_{1}^{2} y} y^{\theta_{1}^{2} / \sigma_{1}^{2}} \cdot \mathbb{1}_{(0,1]}(y),
$$

where $f_{Y}(y)$ is the probability density function of $Y$. The distribution of $\mathbb{h}_{1}$ can be shown to be

$$
f_{\mathrm{h}_{1}}(h)=\Phi_{1} h^{\left(\theta_{1}^{2}-\sigma_{1}^{2}\right) / \sigma_{1}^{2}} \cdot \mathbb{1}_{\left[0, B_{1}\right)}(h),
$$

where $\Phi_{1}:=\frac{\theta_{1}^{2}}{\sigma_{1}^{2}}\left(\frac{1}{B_{1}}\right)^{\theta_{1}^{2} / \sigma_{1}^{2}}$, and

$$
B_{1}:=\frac{e^{-\alpha(D-z)} a_{d}^{2}}{2 \theta_{1}^{2}(D-z)^{2}} .
$$




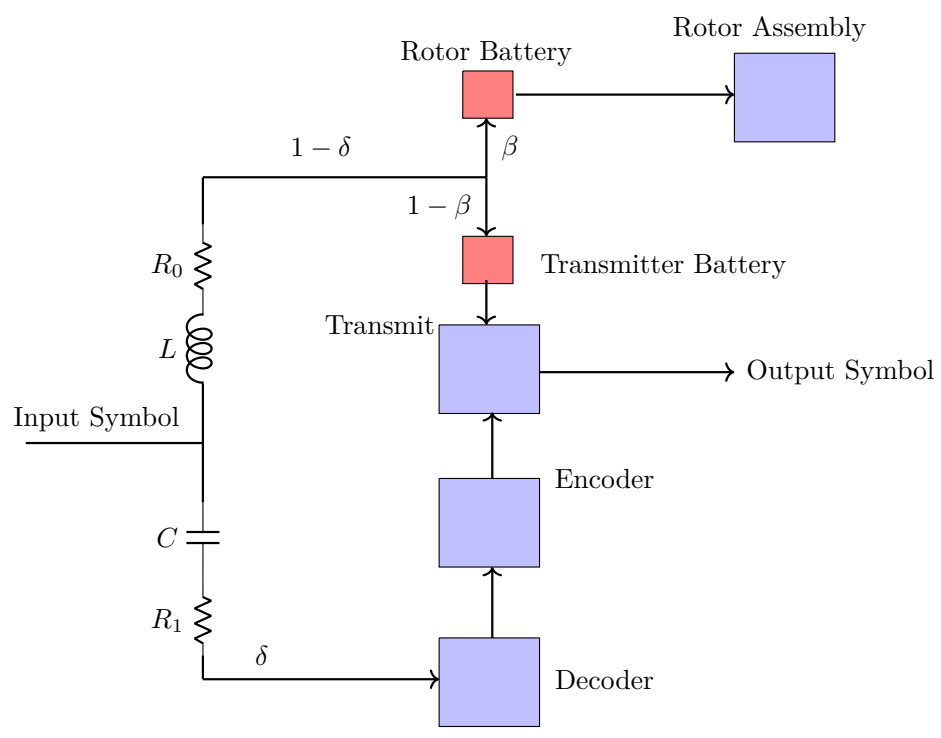

Fig. 2. This figure depicts the architecture of an energy-harvesting decode-and-forward relay.

Similarly, it can be shown that

$$
f_{\mathfrak{h}_{0}}(h)=\Phi_{0} h^{\left(\theta_{0}^{2}-\sigma_{0}^{2}\right) / \sigma_{0}^{2}} \cdot \mathbb{1}_{\left[0, B_{0}\right)}(h),
$$

where $\sigma_{0}$ is the standard deviation of angular pointing error in Channel $0, \Phi_{0}:=\frac{\theta_{0}^{2}}{\sigma_{0}^{2}}\left(\frac{1}{B_{0}}\right)^{\theta_{0}^{2} / \sigma_{0}^{2}}$, and $B_{0}:=\frac{e^{-\alpha z} a_{r}^{2}}{2 \theta_{0}^{2} z^{2}}$.

\section{Simultaneous Lightwave Information and Power Transfer (SLIPT) Receiver Architecture}

Here, we consider a short description of the SLIPT architecture for use on an energy harvesting UAV based relay. A short description of the SLIPT architecture will be given in this section, and the interested readers may refer to [28] for more details on this topic.

There are two ways that the received laser energy can be used for energy harvesting. One is the time splitting technique in which a portion of pulse time is allocated for energy harvesting, and the remaining pulse time is used for data transmission from source to relay. The second technique involves a power splitter that actually splits power between decoding and energy harvesting channels. One example of the latter technique is shown in the block diagram in Fig. 2, where the RC circuit diverts a fraction $\delta$ of received energy (alternating current component of the signal) to the decoding block, and the direct current component $(1-\delta)$ is filtered by the RL circuit to the energy harvesting block (batteries, transmit and rotor blocks). A noncoherent modulation scheme—such as on-off keying (OOK)—already contains a minimum amount of DC component that can be used for energy harvesting purpose. The harvested power, which 
is $(1-\delta) P_{r}$-where $P_{r}$ is the power received at the relay-is further subdivided by a power splitter into fractions $\beta$ (used to charge the rotor battery) and $1-\beta$ (used to charge transmitter battery) as shown in the block diagram. Thus, the power going to the rotor is the component $(1-\delta) \beta P_{r}$, and the remaining energy $(1-\delta)(1-\beta) P_{r}$ is reserved for symbol transmission to the destination.

In our current study, we have assumed a power splitting mechanism for diving energy between each of the decoding and the energy harvesting branches. We assume that the system designer has access to mirror and prism based power splitters that can split laser power in any ratio [29].

\section{Performance Metrics of Decode-And-Forward Relay}

In this section, we derive the performance metrics of the end-to-end link-the maximum achievable rate or ergodic capacity, the outage probability and the error probability-as a function of power split factors $\delta$ and $\beta$. Without loss of generality, we assume that received signal generates the current $i=\eta P_{r}$ in the photodetector where $P_{r}$ is the received power, and $\eta$ is the responsivity of the detector. Without loss of generality, we assume that $\eta=1$ in order to simplify the resulting analysis.

\section{A. Maximum Achievable Rate}

The maximum achievable rate, or the ergodic capacity, of the end-to-end link is the minimum of the individual ergodic capacities of each of the two hops or links:

$$
\mathcal{C}\left(h_{0}, h_{1}\right)=\min \left(\mathcal{C}_{0}\left(h_{0}\right), \mathcal{C}_{1}\left(h_{0}, h_{1}\right)\right)
$$

where $\mathcal{C}_{0}$ and $\mathcal{C}_{1}$ are the individual ergodic capacities of the S-R and R-D channels, respectively.

$$
\begin{aligned}
\mathcal{C}_{0}\left(h_{0}\right) & =\ln \left(1+\frac{\left(\delta P_{t} h_{0}\right)^{2}}{\sigma_{n}^{2}}\right) \\
\mathcal{C}_{1}\left(h_{0}, h_{1}\right) & =\ln \left(1+\frac{\left((1-\beta)(1-\delta) P_{t} h_{0} h_{1}\right)^{2}}{\sigma_{n}^{2}}\right) .
\end{aligned}
$$

The ergodic channel capacity of the end-to-end link is given by

$$
\begin{aligned}
\mathcal{C} & =\int_{0}^{B_{0}} \int_{0}^{B_{1}} \mathcal{C}\left(h_{0}, h_{1}\right) f\left(h_{1}\right) f\left(h_{0}\right) d h_{1} d h_{0} \\
& =\int_{0}^{B_{0}} \int_{0}^{B_{1}} \min \left[\ln \left(1+\frac{\left(\delta P_{t} h_{0}\right)^{2}}{\sigma_{n}^{2}}\right), \ln \left(1+\frac{\left((1-\beta)(1-\delta) P_{t} h_{0} h_{1}\right)^{2}}{\sigma_{n}^{2}}\right)\right] \Phi_{1} h_{1}^{\theta_{1}^{2} / \sigma_{1}^{2}-1} \Phi_{0} h_{0}^{\theta_{0}^{2} / \sigma_{0}^{2}-1} d h_{1} d h_{0}
\end{aligned}
$$


Equation (14) can be simplified further by noting that at a specific value of $h_{1}$, which we denote by $h_{1}^{*}$, we have that

$$
\begin{aligned}
& \ln \left(1+\frac{\left(\delta P_{t} h_{0}\right)^{2}}{\sigma_{n}^{2}}\right)=\ln \left(1+\frac{\left((1-\beta)(1-\delta) P_{t} h_{0} h_{1}^{*}\right)^{2}}{\sigma_{n}^{2}}\right) \\
& \Longrightarrow \delta^{2}=(1-\beta)^{2}(1-\delta)^{2}\left(h_{1}^{*}\right)^{2} \Longrightarrow h_{1}^{*}=\frac{\delta}{(1-\beta)(1-\delta)}
\end{aligned}
$$

Thus, (14) may be rewritten as

$$
\begin{aligned}
& \mathcal{C}=\int_{0}^{B_{0}} \int_{0}^{\min \left(h_{1}^{*}, B_{1}\right)} \ln \left(1+\frac{\left((1-\beta)(1-\delta) P_{t} h_{0} h_{1}\right)^{2}}{\sigma_{n}^{2}}\right) \Phi_{1} h_{1}^{\theta_{1}^{2} / \sigma_{1}^{2}-1} \Phi_{0} h_{0}^{\theta_{0}^{2} / \sigma_{0}^{2}-1} d h_{1} d h_{0} \\
& +\int_{0}^{B_{0}} \int_{\min \left(h_{1}^{*}, B_{1}\right)}^{B_{1}} \ln \left(1+\frac{\left(\delta P_{t} h_{0}\right)^{2}}{\sigma_{n}^{2}}\right) \Phi_{1} h_{1}^{\theta_{1}^{2} / \sigma_{1}^{2}-1} \Phi_{0} h_{0}^{\theta_{0}^{2} / \sigma_{0}^{2}-1} d h_{1} d h_{0} .
\end{aligned}
$$

In order to simplify (16), we first show that

$$
\ln (x) \approx \alpha\left(x^{\frac{1}{\alpha}}-1\right)
$$

for any $x>0$ and for some $\alpha \gg 1$.

\section{1) Proof of Equation (17)}

The Taylor series expansion of a function $f(x)$ is given by

$$
f(x)=\sum_{n=0}^{\infty} \frac{f^{(n)}(b)}{n !}(x-b)^{n}
$$

where $f^{(n)}(\cdot)$ is the $n$th derivative of $f(\cdot)$ and $b$ is any real number. Setting $f(x)=x^{\frac{1}{\alpha}}$ and $b=1$, we have that

$$
\begin{aligned}
x^{\frac{1}{\alpha}} & =1+\frac{1}{1 !} \frac{1}{\alpha}(x-1)+\frac{1}{2 !} \frac{1}{\alpha}\left(\frac{1}{\alpha}-1\right)(x-1)^{2}+\frac{1}{3 !} \frac{1}{\alpha}\left(\frac{1}{\alpha}-1\right)\left(\frac{1}{\alpha}-2\right)(x-1)^{3}+\ldots \\
\alpha\left(x^{\frac{1}{\alpha}}-1\right) & =(x-1)+\frac{1}{2 !}\left(\frac{1}{\alpha}-1\right)(x-1)^{2}+\frac{1}{3 !}\left(\frac{1}{\alpha}-1\right)\left(\frac{1}{\alpha}-2\right)(x-1)^{3}+\ldots \\
\lim _{\alpha \rightarrow \infty} \alpha\left(x^{\frac{1}{\alpha}}-1\right) & =(x-1)-\frac{(x-1)^{2}}{2}+\frac{(x-1)^{3}}{3}-\cdots=\ln (x) .
\end{aligned}
$$

Thus, (16) may be rewritten as

$$
\mathcal{C} \approx \int_{0}^{B_{0}} \int_{0}^{\min \left(h_{1}^{*}, B_{1}\right)}\left(\alpha\left(1+\frac{\left((1-\beta)(1-\delta) P_{t} h_{0} h_{1}\right)^{2}}{\sigma_{n}^{2}}\right)^{\frac{1}{\alpha}}-\alpha\right) \Phi_{1} h_{1}^{\theta_{1}^{2} / \sigma_{1}^{2}-1} \Phi_{0} h_{0}^{\theta_{0}^{2} / \sigma_{0}^{2}-1} d h_{1} d h_{0}
$$




$$
+\int_{0}^{B_{0}} \int_{\min \left(h_{1}^{*}, B_{1}\right)}^{B_{1}}\left(\alpha\left(1+\frac{\left(\delta P_{t} h_{0}\right)^{2}}{\sigma_{n}^{2}}\right)^{\frac{1}{\alpha}}-\alpha\right) \Phi_{1} h_{1}^{\theta_{1}^{2} / \sigma_{1}^{2}-1} \Phi_{0} h_{0}^{\theta_{0}^{2} / \sigma_{0}^{2}-1} d h_{1} d h_{0}
$$

Through the binomial approximation, the quantity $\left(1+\frac{\left((1-\beta)(1-\delta) P_{t} h_{0} h_{1}\right)^{2}}{\sigma_{n}^{2}}\right)^{\frac{1}{\alpha}} \approx 1+\frac{1}{\alpha} \frac{\left((1-\beta)(1-\delta) P_{t} h_{0} h_{1}\right)^{2}}{\sigma_{n}^{2}}$ when $\frac{\left((1-\beta)(1-\delta) P_{t} h_{0} h_{1}\right)^{2}}{\sigma_{n}^{2}} \leq 1$ and $\frac{1}{\alpha} \frac{\left((1-\beta)(1-\delta) P_{t} h_{0} h_{1}\right)^{2}}{\sigma_{n}^{2}} \ll 1$. The condition $\frac{\left((1-\beta)(1-\delta) P_{t} h_{0} h_{1}\right)^{2}}{\sigma_{n}^{2}} \leq 1$ implies $h_{1} \leq \frac{\sigma_{n}}{(1-\beta)(1-\delta) P_{t} h_{0}}$. Let us now define the quantity

$$
h_{1}^{\star}:=\frac{\sigma_{n}}{(1-\beta)(1-\delta) P_{t} h_{0}} \approx \frac{\sigma_{n}}{(1-\beta)(1-\delta) P_{t} B_{0}}
$$

where the approximation in (22) holds loosely for $1<\frac{\theta_{0}^{2}}{\sigma_{0}^{2}}<2$, and the approximation gets better for larger values of the ratio $\frac{\theta_{0}^{2}}{\sigma_{0}^{2}}$ compared to 1 . We will use this approximation in 27 in order to arrive at a closed-form expression of end-to-end ergodic capacity.

Thus, we have that

$$
\begin{aligned}
& \left(1+\frac{\left((1-\beta)(1-\delta) P_{t} h_{0} h_{1}\right)^{2}}{\sigma_{n}^{2}}\right)^{\frac{1}{\alpha}} \approx 1+\frac{1}{\alpha} \frac{\left((1-\beta)(1-\delta) P_{t} h_{0} h_{1}\right)^{2}}{\sigma_{n}^{2}}, 0<h_{1}<h_{1}^{\star} \\
& \left(1+\frac{\left((1-\beta)(1-\delta) P_{t} h_{0} h_{1}\right)^{2}}{\sigma_{n}^{2}}\right)^{\frac{1}{\alpha}} \approx\left(\frac{\left((1-\beta)(1-\delta) P_{t} h_{0} h_{1}\right)^{2}}{\sigma_{n}^{2}}\right)^{\frac{1}{\alpha}}+\frac{1}{\alpha}\left(\frac{\left((1-\beta)(1-\delta) P_{t} h_{0} h_{1}\right)^{2}}{\sigma_{n}^{2}}\right)^{\frac{1}{\alpha}-1}, h_{1}>h_{1}^{\star}
\end{aligned}
$$

and

$$
\begin{aligned}
& \left(1+\frac{\left(\delta P_{t} h_{0}\right)^{2}}{\sigma_{n}^{2}}\right)^{\frac{1}{\alpha}} \approx 1+\frac{1}{\alpha} \frac{\left(\delta P_{t} h_{0}\right)^{2}}{\sigma_{n}^{2}}, 0<h_{0}<h_{0}^{\star} \\
& \left(1+\frac{\left(\delta P_{t} h_{0}\right)^{2}}{\sigma_{n}^{2}}\right)^{\frac{1}{\alpha}} \approx\left(\frac{\left(\delta P_{t} h_{0}\right)^{2}}{\sigma_{n}^{2}}\right)^{\frac{1}{\alpha}}+\frac{1}{\alpha}\left(\frac{\left(\delta P_{t} h_{0}\right)^{2}}{\sigma_{n}^{2}}\right)^{\frac{1}{\alpha}-1}, h_{0}>h_{0}^{*}
\end{aligned}
$$

where $h_{0}^{\star}:=\frac{\sigma_{n}}{\delta P_{t}}$. Denoting the approximate value of $\mathcal{C}$ by $\tilde{\mathcal{C}}$, we can now write (21) approximately as

$$
\begin{aligned}
\mathcal{C} & \approx \tilde{\mathcal{C}}=\int_{0}^{B_{0}} \int_{0}^{\min \left(h_{1}^{\star}, h_{1}^{*}, B_{1}\right)} \frac{\left((1-\beta)(1-\delta) P_{t} h_{0} h_{1}\right)^{2}}{\sigma_{n}^{2}} \Phi_{1} h_{1}^{\theta_{1}^{2} / \sigma_{1}^{2}-1} \Phi_{0} h_{0}^{\theta_{0}^{2} / \sigma_{0}^{2}-1} d h_{1} d h_{0} \\
& +\int_{0}^{B_{0}} \int_{\min \left(h_{1}^{\star}, h_{1}^{*}, B_{1}\right)}^{\min \left(h_{1}^{*}, B_{1}\right)}\left(\alpha\left(\frac{\left((1-\beta)(1-\delta) P_{t} h_{0} h_{1}\right)^{2}}{\sigma_{n}^{2}}\right)^{\frac{1}{\alpha}}+\left(\frac{\left((1-\beta)(1-\delta) P_{t} h_{0} h_{1}\right)^{2}}{\sigma_{n}^{2}}\right)^{\frac{1}{\alpha}-1}-\alpha\right) \Phi_{1} h_{1}^{\theta_{1}^{2} / \sigma_{1}^{2}-1} \Phi_{0} h_{0}^{\theta_{0}^{2} /} \\
& +\int_{\min \left(h_{1}^{*}, B_{1}\right)}^{B_{1}} \Phi_{1} h_{1}^{\theta_{1}^{2} / \sigma_{1}^{2}-1} d h_{1}\left(\int_{0}^{\min \left(h_{0}^{\star}, B_{0}\right)}\left(\frac{\left(\delta P_{t} h_{0}\right)^{2}}{\sigma_{n}^{2}}\right) \Phi_{0} h_{0}^{\theta_{0}^{2} / \sigma_{0}^{2}-1} d h_{0}\right. \\
& \left.+\int_{\min \left(h_{0}^{\star}, B_{0}\right)}^{B_{0}}\left(\alpha\left(\frac{\left(\delta P_{t} h_{0}\right)^{2}}{\sigma_{n}^{2}}\right)^{\frac{1}{\alpha}}+\left(\frac{\left(\delta P_{t} h_{0}\right)^{2}}{\sigma_{n}^{2}}\right)^{\frac{1}{\alpha}-1}-\alpha\right) \Phi_{0} h_{0}^{\theta_{0}^{2} / \sigma_{0}^{2}-1} d h_{0}\right)
\end{aligned}
$$

Equation 27) contains straightforward integrals that are easy to compute. After a number of steps, a 
closed-form solution to (27) is produced below.

$$
\begin{aligned}
& \tilde{\mathcal{C}}=\Phi_{0} \Phi_{1}\left(\frac{(1-\beta)(1-\delta) P_{t}}{\sigma_{n}}\right)^{2} \frac{\sigma_{1}^{2}}{\left(\theta_{1}^{2}+2 \sigma_{1}^{2}\right)}\left(\min \left(h_{1}^{\star}, h_{1}^{*}, B_{1}\right)\right)^{\frac{\theta_{1}^{2}+2 \sigma_{1}^{2}}{\sigma_{1}^{2}}}\left(\frac{\sigma_{0}^{2}}{\left(\theta_{0}^{2}+2 \sigma_{0}^{2}\right)}\right) B_{0}^{\frac{\theta_{0}^{2}+2 \sigma_{0}^{2}}{\sigma_{0}^{2}}} \\
& +\Phi_{0} \Phi_{1} \alpha\left(\frac{(1-\beta)(1-\delta) P_{t}}{\sigma_{n}}\right)^{2}\left(\frac{\alpha \sigma_{1}^{2}}{2 \sigma_{1}^{2}+\alpha \theta_{1}^{2}}\right)\left(\frac{\sigma_{0}^{2}}{\theta_{0}^{2}+2 \sigma_{0}^{2}}\right)\left(\left(\min \left(h_{1}^{*}, B_{1}\right)\right)^{\frac{2 \sigma_{1}^{2}+\alpha \theta_{1}^{2}}{\alpha \sigma_{1}^{2}}}-\left(\min \left(h_{1}^{\star}, h_{1}^{*}, B_{1}\right)\right)^{\frac{2 \sigma_{1}^{2}+\alpha \theta_{1}^{2}}{\alpha \sigma_{1}^{2}}}\right) \\
& \times B_{0}^{\frac{\theta_{0}^{2}+2 \sigma_{0}^{2}}{\sigma_{0}^{2}}}+\Phi_{0} \Phi_{1}\left(\left(\frac{(1-\beta)(1-\delta) P_{t}}{\sigma_{n}}\right)^{2}\right)^{\frac{1-\alpha}{\alpha}}\left(\frac{\alpha \sigma_{1}^{2}}{2(1-\alpha) \sigma_{1}^{2}+\theta_{1}^{2} \alpha}\right)\left(\frac{\alpha \sigma_{0}^{2}}{2(1-\alpha) \sigma_{0}^{2}+\theta_{0}^{2} \alpha}\right) \\
& \times\left(\left(\min \left(h_{1}^{*}, B_{1}\right)\right)^{\frac{2(1-\alpha) \sigma_{1}^{2}+\alpha \theta_{1}^{2}}{\alpha \sigma_{1}^{2}}}-\left(\min \left(h_{1}^{\star}, h_{1}^{*}, B_{1}\right)\right)^{\frac{2(1-\alpha) \sigma_{1}^{2}+\alpha \theta_{1}^{2}}{\alpha \sigma_{1}^{2}}}\right) B_{0}^{\frac{2(1-\alpha) \sigma_{0}^{2}+\alpha \theta_{0}^{2}}{\alpha \sigma_{0}^{2}}}-\Phi_{0} \Phi_{1} \alpha \frac{\sigma_{0}^{2} \sigma_{1}^{2}}{\theta_{0}^{2} \theta_{1}^{2}} \\
& \times\left(\left(\min \left(h_{1}^{*}, B_{1}\right)\right)^{\frac{\theta_{1}^{2}}{\sigma_{1}^{2}}}-\left(\min \left(h_{1}^{\star}, h_{1}^{*}, B_{1}\right)\right)^{\frac{\theta_{1}^{2}}{\sigma_{1}^{2}}}\right) B_{0}^{\frac{\theta_{0}^{2}}{\sigma_{0}^{2}}}+\Phi_{1} \frac{\sigma_{1}^{2}}{\theta_{1}^{2}}\left(B_{1}^{\frac{\theta_{1}^{2}}{\sigma_{1}^{2}}}-\left(\min \left(h_{1}^{*}, B_{1}\right)\right)^{\frac{\theta_{1}^{2}}{\sigma_{1}^{2}}}\right) \\
& \times \Phi_{0}\left[\left(\frac{\delta P_{t}}{\sigma_{n}}\right)^{2}\left(\frac{\sigma_{0}^{2}}{\theta_{0}^{2}+2 \sigma_{0}^{2}}\right)\left(\min \left(h_{0}^{\star}, B_{0}\right)\right)^{\frac{\theta_{0}^{2}+2 \sigma_{0}^{2}}{\sigma_{0}^{2}}}+\alpha\left(\frac{\delta P_{t}}{\sigma_{n}}\right)^{\frac{2}{\alpha}}\left(\frac{\alpha \sigma_{0}^{2}}{2 \sigma_{0}^{2}+\alpha \theta_{0}^{2}}\right)\right. \\
& \times\left(B_{0}^{\frac{2 \sigma_{0}^{2}+\theta_{0}^{2} \alpha}{\alpha \sigma_{0}^{2}}}-\left(\min \left(h_{0}^{\star}, B_{0}\right)\right)^{\frac{2 \sigma_{0}^{2}+\theta_{0}^{2} \alpha}{\alpha \sigma_{0}^{2}}}\right)+\left(\frac{\delta P_{t}}{\sigma_{n}}\right)^{2}\left(\frac{\alpha \sigma_{0}^{2}}{2(1-\alpha) \sigma_{0}^{2}+\theta_{0}^{2} \alpha}\right) \\
& \left.\times\left(B_{0}^{\frac{2(1-\alpha) \sigma_{0}^{2}+\theta_{0}^{2} \alpha}{\alpha \sigma_{0}^{2}}}-\left(\min \left(h_{0}^{\star}, B_{0}\right)\right)^{\frac{2(1-\alpha) \sigma_{0}^{2}+\theta_{0}^{2} \alpha}{\alpha \sigma_{0}^{2}}}\right)-\alpha \frac{\sigma_{0}^{2}}{\theta_{0}^{2}}\left(B_{0}^{\frac{\theta_{0}^{2}}{\sigma_{0}^{2}}}-\left(\min \left(h_{0}^{\star}, B_{0}\right)\right)^{\frac{\theta_{0}^{2}}{\sigma_{0}^{2}}}\right)\right] .
\end{aligned}
$$

In order to quantify the approximation error between $\mathcal{C}$ and $\tilde{\mathcal{C}}$, we use the normalized mean-square error (NMSE) criterion. The NMSE is defined as the ratio of $L^{2}$-norm of the error to the $L^{2}$-norm of original quantity as follows.

$$
\mathrm{NMSE}:=\frac{\int_{0}^{1}[\mathcal{C}(\delta)-\tilde{\mathcal{C}}(\delta)]^{2} d \delta}{\int_{0}^{1} \mathcal{C}(\delta)^{2} d \delta}
$$

Figure 3 shows the normalized mean-square error as a function of ratio $\frac{\theta_{0}^{2}}{\sigma_{0}^{2}}$ for two values of noise standard deviation $\sigma_{n}$. We note that the approximation error decreases monotonically as $\frac{\theta_{0}^{2}}{\sigma_{0}^{2}}$ grows large compared to 1 .

\section{B. Outage Probability}

The outage event will not occur at the relay if the SNR at the relay stays above a certain predefined threshold $\Upsilon_{\text {th }}$ :

$$
\operatorname{Pr}\left(\mathcal{O}_{0}^{\complement}\right):=\operatorname{Pr}\left(\left\{\frac{\left(\delta P_{t} \mathfrak{h}_{0}\right)^{2}}{\sigma_{n}^{2}}>\Upsilon_{\text {th }}\right\}\right)=\operatorname{Pr}\left(\left\{\mathfrak{h}_{0}^{2}>\Upsilon_{\text {th }} \frac{\sigma_{n}^{2}}{\delta^{2} P_{t}^{2}}\right\}\right) .
$$




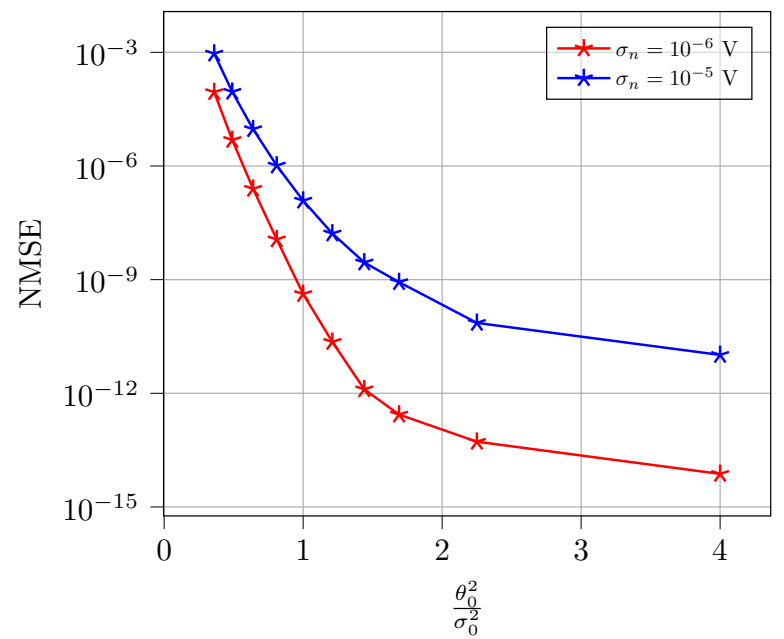

Fig. 3. This figure shows the normalized mean-square error as a function of the factor $\theta_{0}^{2} / \sigma_{0}^{2}$ for two values of thermal noise standard deviation $\sigma_{n}$. The total link distance $D=4000 \mathrm{~m}$, transmitted power $P_{t}=50 \mathrm{~W}$, the beamwidth $\theta_{1}$ and angular error standard deviation $\sigma_{1}$ in Channel 1 are $2 \mathrm{mrad}$ and $1 \mathrm{mrad}$, respectively. The distance $z=2000 \mathrm{~m}$, the factor $\beta=0$, the aperture radius for relay $a_{r}$ and the destination $a_{d}$ are $0.2 \mathrm{~m}$ and $1 \mathrm{~m}$, respectively.

It can be shown that the cumulative distribution function of $\mathbb{h}_{0}^{2}$ and $\mathbb{h}_{1}^{2}$ is

$$
\begin{aligned}
& F_{\mathrm{h}_{0}^{2}}(y)=\left(\frac{y}{\mathcal{B}_{0}}\right)^{\theta_{0}^{2} /\left(2 \sigma_{0}^{2}\right)} \cdot \mathbb{1}_{\left[0, \mathcal{B}_{0}\right)}(y) \\
& F_{\mathrm{h}_{1}^{2}}(y)=\left(\frac{y}{\mathcal{B}_{1}}\right)^{\theta_{1}^{2} /\left(2 \sigma_{1}^{2}\right)} \cdot \mathbb{1}_{\left[0, \mathcal{B}_{1}\right)}(y)
\end{aligned}
$$

where $\mathcal{B}_{0}:=\frac{\exp (-2 \alpha z) a_{r}^{4}}{4 \theta_{0}^{4} z^{4}}$, and $\mathcal{B}_{1}:=\frac{\exp (-2 \alpha(D-z)) a_{d}^{4}}{4 \theta_{1}^{4}(D-z)^{4}}$. Thus, we have that

$$
\operatorname{Pr}\left(\mathcal{O}_{0}^{\complement}\right)=1-\left(\frac{\Upsilon_{\mathrm{th}} \sigma_{n}^{2}}{\delta^{2} P_{t}^{2} \mathcal{B}_{0}}\right)^{\theta_{0}^{2} /\left(2 \sigma_{0}^{2}\right)} \cdot \mathbb{1}_{\left[0, \mathcal{B}_{0}^{\prime}\right)}\left(\Upsilon_{\mathrm{th}}\right)
$$

where $\mathcal{B}_{0}^{\prime}:=\frac{\mathcal{B}_{0} \delta^{2} P_{t}^{2}}{\sigma_{n}^{2}}$.

The outage will not occur for the R-D link if

$$
\begin{aligned}
& \operatorname{Pr}\left(\mathcal{O}_{1}^{\complement}\right)=\operatorname{Pr}\left(\left\{\frac{(1-\beta)^{2}(1-\delta)^{2} P_{t}^{2} \mathfrak{h}_{0}^{2} \mathfrak{h}_{1}^{2}}{\sigma_{n}^{2}}>\Upsilon_{\text {th }}\right\}\right)=\operatorname{Pr}\left(\left\{\mathfrak{h}_{0}^{2} \mathbb{h}_{1}^{2}>\Upsilon_{\text {th }} \frac{\sigma_{n}^{2}}{(1-\beta)^{2}(1-\delta)^{2} P_{t}^{2}}\right\}\right) \\
& =\operatorname{Pr}\left(\left\{\exp \left(-\left(X_{1}+X_{2}\right)\right)>\Upsilon_{\text {th }} \frac{\sigma_{n}^{2}}{(1-\beta)^{2}(1-\delta)^{2} P_{t}^{2}}\left(\frac{2 \theta_{1} \theta_{0} z(D-z)}{a_{r} a_{d}}\right)^{4} \exp (2 \alpha D)\right\}\right)
\end{aligned}
$$

where $X_{1}:=\frac{R_{0}^{2}}{\theta_{0}^{2} z^{2}}$ and $X_{2}:=\frac{R_{1}^{2}}{\theta_{1}^{2}(D-z)^{2}}$ are exponential random variables with means $\mathrm{E}\left[X_{1}\right]=\frac{2 \sigma_{0}^{2}}{\theta_{0}^{2}}$ and $\mathrm{E}\left[X_{2}\right]=\frac{2 \sigma_{1}^{2}}{\theta_{1}^{2}}$. Let us define $Y=X_{1}+X_{2}$ Then,

$$
\operatorname{Pr}\left(\mathcal{O}_{1}^{\complement}\right)=\operatorname{Pr}\left(\left\{Y \leq \ln \left(\frac{1}{\Upsilon_{\text {th }}^{\prime}}\right)\right\}\right)
$$


where $Y$ is a hypoexponentially distributed random variable whose cumulative distribution function is

$$
\operatorname{Pr}(\{Y \leq y\})=1-\frac{\sigma_{1}^{2} \sigma_{0}^{2}}{\left(\theta_{1}^{2} \sigma_{0}^{2}-\theta_{0}^{2} \sigma_{1}^{2}\right)}\left[\frac{\theta_{1}^{2}}{\sigma_{1}^{2}} \exp \left(-\frac{\theta_{0}^{2}}{2 \sigma_{0}^{2}} y\right)-\frac{\theta_{0}^{2}}{\sigma_{0}^{2}} \exp \left(-\frac{\theta_{1}^{2}}{2 \sigma_{1}^{2}} y\right)\right]
$$

for $y>0$. Thus, we have that

$$
\operatorname{Pr}\left(\mathcal{O}_{1}^{\complement}\right)=1-\frac{\sigma_{1}^{2} \sigma_{0}^{2}}{\left(\theta_{1}^{2} \sigma_{0}^{2}-\theta_{0}^{2} \sigma_{1}^{2}\right)}\left[\frac{\theta_{1}^{2}}{\sigma_{1}^{2}}\left(\Upsilon_{\text {th }}^{\prime}\right)^{\theta_{0}^{2} /\left(2 \sigma_{0}^{2}\right)}-\frac{\theta_{0}^{2}}{\sigma_{0}^{2}}\left(\Upsilon_{\text {th }}^{\prime}\right)^{\theta_{1}^{2} /\left(2 \sigma_{1}^{2}\right)}\right] \cdot \mathbb{1}_{(0,1)}\left(\Upsilon_{\text {th }}^{\prime}\right)
$$

where

$$
\Upsilon_{\text {th }}^{\prime}:=\Upsilon_{\text {th }} \frac{\sigma_{n}^{2}}{(1-\beta)^{2}(1-\delta)^{2} P_{t}^{2}}\left(\frac{2 \theta_{1} \theta_{0} z(D-z)}{a_{r} a_{d}}\right)^{4} \exp (2 \alpha D)
$$

Finally, the probability of outage for the end-to-end link is given by

$$
\operatorname{Pr}(\mathcal{O})=1-\operatorname{Pr}\left(\mathcal{O}_{0}^{\complement}\right) \operatorname{Pr}\left(\mathcal{O}_{1}^{\complement}\right)
$$

\section{Error Probability}

The error event for a dual-hop relay occurs if there is an error in decision either after the first hop (at the relay R) or after the second hop (at destination D), but not both. Let us denote the error event at the relay by $\mathcal{E}_{0}$. For OOK signaling, the probability of error at the relay is

$$
\operatorname{Pr}\left(\mathcal{E}_{0} \mid h_{0}\right)=Q\left(\frac{\delta P_{t} h_{0}}{2 \sigma_{n}}\right)
$$

As discussed before, the relay transmits $P_{t}^{(\mathrm{R})}=(1-\beta)(1-\delta) P_{t} \mathrm{~h}_{0}$ Watts of power to the destination. The error event at the destination is denoted by $\mathcal{E}_{1}$. The conditional probability of error at the destination is

$$
\operatorname{Pr}\left(\mathcal{E}_{1} \mid h_{0}\right)=\int_{0}^{B_{1}} Q\left(\frac{(1-\beta)(1-\delta) P_{t} h_{0} h_{1}}{2 \sigma_{n}}\right) f\left(h_{1}\right) d h_{1}
$$

Since the events $\mathcal{E}_{0}$ and $\mathcal{E}_{1}$ are conditionally independent (conditioned on $h_{0}$ ), we have that the conditional error probability of the end-to-end link is

$$
\operatorname{Pr}\left(\mathcal{E} \mid h_{0}\right)=\operatorname{Pr}\left(\mathcal{E}_{0} \mid h_{0}\right)+\operatorname{Pr}\left(\mathcal{E}_{1} \mid h_{0}\right)-2 \operatorname{Pr}\left(\mathcal{E}_{0} \cap \mathcal{E}_{1} \mid h_{0}\right)=\operatorname{Pr}\left(\mathcal{E}_{0} \mid h_{0}\right)+\operatorname{Pr}\left(\mathcal{E}_{1} \mid h_{0}\right)-2 \operatorname{Pr}\left(\mathcal{E}_{0} \mid h_{0}\right) \operatorname{Pr}\left(\mathcal{E}_{1} \mid h_{0}\right)
$$


where the last equality is true because the events $\mathcal{E}_{\mathrm{R}}$ and $\mathcal{E}_{\mathrm{D}}$ are statistically (conditionally) independent of each other. The final expression of probability of error is

$$
\begin{aligned}
\operatorname{Pr}(\mathcal{E}) & =\int_{0}^{B_{0}} \operatorname{Pr}\left(\mathcal{E} \mid h_{0}\right) f\left(h_{0}\right) d h_{0} \\
& =\int_{0}^{B_{0}} Q\left(\frac{\delta P_{t} h_{0}}{2 \sigma_{n}}\right) \Phi_{0} h_{0}^{\theta_{0}^{2} / \sigma_{0}^{2}-1} d h_{0}+\int_{0}^{B_{0}} \int_{0}^{B_{1}} Q\left(\frac{(1-\beta)(1-\delta) P_{t} h_{0} h_{1}}{2 \sigma_{n}}\right) \Phi_{1} h_{1}^{\theta_{1}^{2} / \sigma_{1}^{2}-1} \Phi_{0} h_{0}^{\theta_{0}^{2} / \sigma_{0}^{2}-1} d h_{1} d h_{0} \\
& -2 \int_{0}^{B_{0}} Q\left(\frac{\delta P_{t} h_{0}}{2 \sigma_{n}}\right) \int_{0}^{B_{1}} Q\left(\frac{(1-\beta)(1-\delta) P_{t} h_{0} h_{1}}{2 \sigma_{n}}\right) \Phi_{1} h_{1}^{\theta_{1}^{2} / \sigma_{1}^{2}-1} \Phi_{0} h_{0}^{\theta_{0}^{2} / \sigma_{0}^{2}-1} d h_{1} d h_{0}
\end{aligned}
$$

\section{ENERGy OptimizATION}

Let us define the average received power at the relay be $P_{r}:=P_{t} \mathrm{E}\left[\mathrm{h}_{0}\right]$, and the harvested power from the received laser as $P_{h}:=\beta(1-\delta) P_{r}$ Watts. Let us denote the power consumed by UAV rotors as $P_{0}$.

\section{A. Optimization: $P_{0} \approx 0$}

Here we assume that the power consumed by the UAV rotors is negligible, i.e., $P_{0} \approx 0$. This implies that $\beta \approx 0$ in this optimization problem, and we only optimize with respect to $\delta$. In this scenario, a telescope that focuses energy on a focal plane array acts as an aperture at the relay.

\section{1) Maximization of Maximum Achievable Rate}

$$
\begin{array}{ll}
\underset{\delta}{\operatorname{maximize}} & \mathcal{C} \\
\text { subject to } & \text { i) } 0<\delta<1 .
\end{array}
$$

In order to solve this optimization problem, we consider the expression of the ergodic capacity given by (14). We note that too large a value of $\delta$ will make the factor $\ln \left(1+\frac{\left((1-\beta)(1-\delta) P_{t} h_{0} h_{1}\right)^{2}}{\sigma_{n}^{2}}\right)$ much smaller than $\ln \left(1+\frac{\left(\delta P_{t} h_{0}\right)^{2}}{\sigma_{n}^{2}}\right)$, and a too small a value of $\delta$ will lead to $\ln \left(1+\frac{\left(\delta P_{t} h_{0}\right)^{2}}{\sigma_{n}^{2}}\right) \ll \ln \left(1+\frac{\left((1-\beta)(1-\delta) P_{t} h_{0} h_{1}\right)^{2}}{\sigma_{n}^{2}}\right)$. In either scenario, the ergodic capacity will be minimized at the extrema $(\delta \approx 0$ or $\delta \approx 1)$ since it is computed based on the minimum of the aforementioned factors. Hence, an optimum value of $\delta$ exists between 0 and 1 that will maximize the ergodic capacity.

The minimum function in the integrand of (14) is maximized when the two arguments become equal:

$$
\ln \left(1+\frac{\left(\delta P_{t} h_{0}\right)^{2}}{\sigma_{n}^{2}}\right)=\ln \left(1+\frac{\left((1-\beta)(1-\delta) P_{t} h_{0} h_{1}\right)^{2}}{\sigma_{n}^{2}}\right)
$$

Substituting $\beta=0$ and solving (46), we obtain an optimum value of $\delta$ by

$$
\frac{\delta P_{t} h_{0}}{\sigma_{n}}=\frac{(1-\delta) P_{t} h_{0} h_{1}}{\sigma_{n}} \Longrightarrow \delta^{*}=\frac{h_{1}}{1+h_{1}}
$$




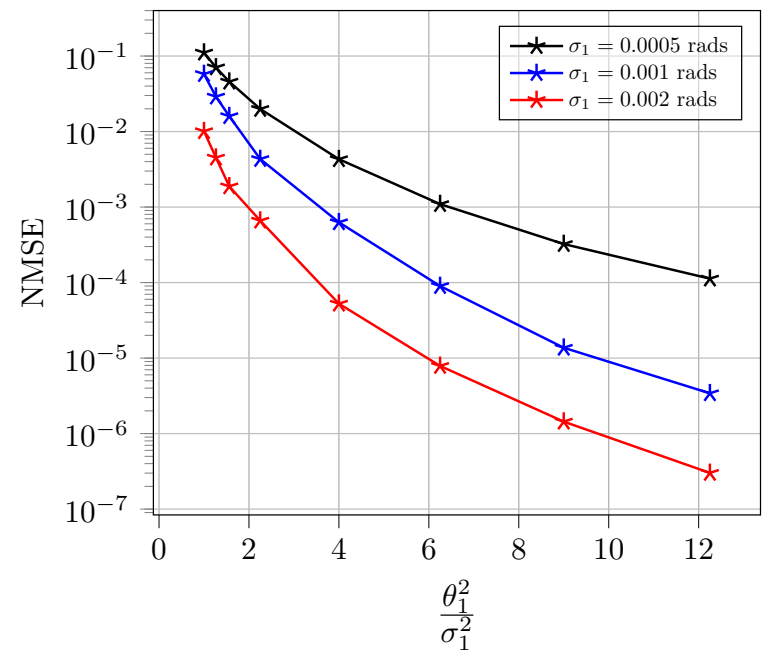

Fig. 4. This figure shows the normalized mean-square error of (Equation (49) as a function of $\theta_{1}^{2} / \sigma_{1}^{2}$. The distance $D=4000 \mathrm{~m}$, transmitted power $P_{t}=50 \mathrm{~W}$, noise standard deviation $\sigma_{n}=1 \times 10^{-7} \mathrm{~V}$, beamwidth angle for Channel $0 \theta_{0}=2$ mrad, angular pointing error standard deviation for Channel $0, \sigma_{0}=1 \mathrm{mrad}$, angular pointing error standard deviation for Channel $1, \sigma_{1}=0.5 \mathrm{mrad}$, distance $z=2000 \mathrm{~m}$, and aperture radii $a_{r}=0.2 \mathrm{~m}$ and $a_{d}=1 \mathrm{~m}$.

which is a function of $h_{1}$. However, when $\theta_{1}^{2} \gg \sigma_{1}^{2}, h_{1}$ takes on the value $B_{1}$ with a high probability. Under this condition, the optimum value of $\delta$ is given by

$$
\delta^{*} \approx \tilde{\delta}:=\frac{B_{1}}{1+B_{1}}
$$

and the normalized mean-square error is computed as

$$
\mathrm{NMSE}=\frac{\left(\delta^{*}-\tilde{\delta}\right)^{2}}{\left(\delta^{*}\right)^{2}} .
$$

Incidentally, we note here that the optimum value of $\delta$ only depends only on the conditions of the channel between the relay and the destination (Channel 1), and is independent of the channel coefficient between the source and the relay. Fig. 4 shows the normalized mean-square error $(\sqrt{49})$ ) as a function of ratio $\frac{\theta_{1}^{2}}{\sigma_{1}^{2}}$.

2) Minimization of Outage/Error Probability

$$
\begin{array}{ll}
\underset{\delta}{\operatorname{minimize}} & f(\delta) \\
\text { subject to } & \text { i) } 0<\delta<1 .
\end{array}
$$

where $f(\delta)$ is either the outage probability or the error probability.

In order to minimize the outage probability, we need to maximize both $\operatorname{Pr}\left(\mathcal{O}_{0}^{\complement}\right)$ and $\operatorname{Pr}\left(\mathcal{O}_{1}^{\complement}\right)$ in Equa- 
tion (40). Maximizing $\operatorname{Pr}\left(\mathcal{O}_{0}^{\complement}\right)$ requires us to maximize $\delta$ (please see Equation (34)). However, in order to maximize $\operatorname{Pr}\left(\mathcal{O}_{1}^{\complement}\right)$, we need to minimize $\Upsilon_{\text {th }}^{\prime}$ which calls for the minimization of $\delta$ in Equation (38). Hence, the optimum $\delta$ should neither be too small, nor too large, for the minimization of outage probability.

The minimization of the outage and error probabilities in terms of $\delta$ is not a straightforward exercise which leads us to explore numerical optimization techniques in order to find the optimum value of $\delta$.

\section{B. Optimization: $P_{r} \geq P_{0}$}

In this setting, we assume that the power $P_{0}$ consumed by the UAV rotors in order to sustain the UAV at a certain height is significant. As there is no external power source, the power $P_{0}$ has to be supplied by the laser beam as well. Here, we assume that a photovoltaic array of appropriate size is mounted on the energy harvesting relay which is used both for energy harvesting as well as communications purpose, and that the total received signal energy $P_{r} \geq P_{0}$. Regarding the energy consumption models for hovering and mobile UAVs, the reader is referred to [30] for a comprehensive study on this topic.

Using a photovoltaic array for both communications and energy harvesting calls for carefully selecting the depletion region of each PN diode in the array: a thicker depletion region leads to a higher quantum efficiency (more energy is harvested), but such a thicker depletion region also slows down the switching response of the diode which leads to a poorer bandwidth [31]. Hence, this trade-off has to be carefully considered before a photovoltaic array is selected for joint communications and energy harvesting.

Alternatively, we may use a hybrid arrangement at the relay aperture where a telescope in conjunction with a photodiode (or a photodiode array) is used for data detection, and a photovoltaic array is used solely for energy harvesting. In this arrangement, a portion of the photovoltaic array may be cut out from its center in order to accomodate the telescope. We assume that the area of the hybrid aperture is larger than the footprint of the beam, and that the area of the aperture is large enough so that the loss in signal energy due to pointing error is negligible [32]. In this scenario, the channel coefficient $\mathbb{h}_{0}$ can be treated as a deterministic quantity which is close to unity. Additionally, this approximation implies that the received average power during pulse time at the relay is $P_{r}=P_{t} \mathrm{E}\left[\mathbb{h}_{0}\right] \approx P_{t}$.

Since a photovoltaic array or the hybrid aperture may be of a much larger area than the telescope aperture area, we assume that the noise power generated by the photovoltaic array is much larger in magnitude than a photodiode receiver. Thus, $\sigma_{n_{0}}^{2} \gg \sigma_{n_{1}}^{2}$ where $\sigma_{n_{0}}^{2}$ is the noise power in S-R link (photovoltaic array and photodiode), and $\sigma_{n_{1}}^{2}$ represents noise power in R-D link (only photodiode receiver). 


\section{1) Ergodic Capacity}

By using the same line of arguments as in Section IV-A the ergodic channel capacity for the end-to-end link-when $\mathrm{h}_{0} \approx 1$-is given by

$$
\begin{aligned}
\mathcal{C} & =\int_{0}^{B_{1}} \min \left[\ln \left(1+\frac{\left(\delta P_{t}\right)^{2}}{\sigma_{n_{0}}^{2}}\right), \ln \left(1+\frac{\left((1-\beta)(1-\delta) P_{t} h_{1}\right)^{2}}{\sigma_{n_{1}}^{2}}\right)\right] \Phi_{1} h_{1}^{\theta_{1}^{2} / \sigma_{1}^{2}-1} d h_{1} \\
& \approx \Phi_{1}\left[\left(\frac{(1-\beta)(1-\delta) P_{t}}{\sigma_{n_{1}}}\right)^{2} \frac{\sigma_{1}^{2}}{\theta_{1}^{2}}\left(\left(\min \left(h_{1}^{\star}, h_{1}^{*}, B_{1}\right)\right)^{\frac{\theta_{1}^{2}}{\sigma_{1}^{2}}}\right)+\alpha\left(\frac{(1-\beta)(1-\delta) P_{t}}{\sigma_{n_{1}}}\right)^{\frac{2}{\alpha}}\left(\frac{\alpha \sigma_{1}^{2}}{2 \sigma_{1}^{2}+\alpha \theta_{1}^{2}}\right)\right. \\
& \times\left(\left(\min \left(h_{1}^{*}, B_{1}\right)\right)^{\frac{2 \sigma_{1}^{2}+\alpha \theta_{1}^{2}}{\alpha \sigma_{1}^{2}}}-\left(\min \left(h_{1}^{\star}, h_{1}^{*}, B_{1}\right)\right)^{\frac{2 \sigma_{1}^{2}+\alpha \theta_{1}^{2}}{\alpha \sigma_{1}^{2}}}\right)+\left(\frac{(1-\beta)(1-\delta) P_{t}}{\sigma_{n_{1}}}\right)^{\frac{2(1-\alpha)}{\alpha}}\left(\frac{\alpha \sigma_{1}^{2}}{2(1-\alpha) \sigma_{1}^{2}+\alpha \theta_{1}^{2}}\right) \\
& \left.\times\left(\left(\min \left(h_{1}^{*}, B_{1}\right)\right)^{\frac{2(1-\alpha) \sigma_{1}^{2}+\alpha \theta_{1}^{2}}{\alpha \sigma_{1}^{2}}}-\left(\min \left(h_{1}^{\star}, h_{1}^{*}, B_{1}\right)\right)^{\frac{2(1-\alpha) \sigma_{1}^{2}+\alpha \theta_{1}^{2}}{\alpha \sigma_{1}^{2}}}\right)+\ln \left(1+\left(\frac{\delta P_{t}}{\sigma_{n_{0}}}\right)^{2}\right) \frac{\sigma_{1}^{2}}{\theta_{1}^{2}}\left(B_{1}^{\frac{\theta_{1}^{2}}{\sigma_{1}^{2}}}-\left(\min \left(h_{1}^{*}, B_{1}\right)\right)^{\frac{\theta_{1}^{2}}{\sigma_{1}^{2}}}\right)\right]
\end{aligned}
$$

where $h_{1}^{*}:=\frac{\sigma_{n_{1}} \delta}{\sigma_{n_{0}}(1-\delta)(1-\beta)}$ and $h_{1}^{\star}:=\frac{\sigma_{n_{1}}}{(1-\delta)(1-\beta) P_{t}}$.

\section{2) Outage Probability}

The probability that there is no outage in the R-D link is

$$
\begin{aligned}
\operatorname{Pr}\left(\mathcal{O}_{1}^{\complement}\right) & =\operatorname{Pr}\left(\left\{\frac{(1-\beta)^{2}(1-\delta)^{2} P_{t}^{2} \mathfrak{h}_{1}^{2}}{\sigma_{n_{1}}^{2}} \geq \Upsilon_{\text {th }}\right\}\right)=\operatorname{Pr}\left(\left\{\mathfrak{h}_{1}^{2} \geq \frac{\Upsilon_{\text {th }} \sigma_{n_{1}}^{2}}{(1-\beta)^{2}(1-\delta)^{2} P_{t}^{2}}\right\}\right) \\
& =1-\left(\Upsilon_{\text {th }}^{\prime}\right)^{\frac{\theta_{1}^{2}}{2 \sigma_{1}^{2}}} \cdot \mathbb{1}_{[0, \psi)}\left(\Upsilon_{\text {th }}\right),
\end{aligned}
$$

where

$$
\Upsilon_{\text {th }}^{\prime}:=\frac{\Upsilon_{\text {th }} \sigma_{n_{1}}^{2}}{(1-\beta)^{2}(1-\delta)^{2} P_{t}^{2} B_{1}},
$$

and $\psi:=\frac{(1-\beta)^{2}(1-\delta)^{2} P_{t}^{2} B_{1}}{\sigma_{n_{1}}^{2}}$. For the S-R link, the channel is assumed to be deterministic, and we are guaranteed that there is no outage in the link if the $\operatorname{SNR} \frac{\left(\delta P_{t}\right)^{2}}{\sigma_{n_{0}}^{2}}$ is greater than the threshold $\Upsilon_{\text {th. }}$ This translates to the following additional condition on $\delta$ in the optimization problem:

$$
\frac{\delta^{2} P_{t}^{2}}{\sigma_{n_{0}}^{2}}>\Upsilon_{\mathrm{th}} \Longrightarrow \delta>\frac{\Upsilon_{\mathrm{th}} \sigma_{n_{0}}}{P_{t}}
$$

Thus, the outage probability of the end-to-end link is

$$
\operatorname{Pr}(\mathcal{O})=1-\operatorname{Pr}\left(\mathcal{O}_{1}^{\complement}\right)=\left(\Upsilon_{\text {th }}^{\prime}\right)^{\frac{\theta_{1}^{2}}{2 \sigma_{1}^{2}}} \cdot \mathbb{1}_{[0, \psi)}\left(\Upsilon_{\text {th }}\right)
$$


under the constraint $\delta>\frac{\Upsilon_{\text {th }} \sigma_{n_{0}}}{P_{t}}$.

\section{3) Optimization Problems}

The goal of this section is to optimize a performance metric (outage probability and the ergodic capacity) under the constraint that $P_{h}>P_{0}$. This constraint guarantees a sustainable operation of the UAV without incurring any downtime. We have that the constraint $P_{h}>P_{0}$ implies $\beta(1-\delta)>\frac{P_{0}}{P_{R}} \approx \frac{P_{0}}{P_{t}}$.

a) Optimization Problem 1:

$$
\begin{array}{ll}
\underset{\delta, \beta}{\operatorname{maximize}} & \mathcal{C} \\
\text { subject to } & \text { i) } 0<\delta<1, \\
& \text { ii) } 0<\beta<1, \\
& \text { iii) } \beta(1-\delta)>\frac{P_{0}}{P_{t}} .
\end{array}
$$

For this optimization problem, an approximate closed form expression for $\beta^{*}$ and $\delta^{*}$ exists. In order to see that, we note the fact that the solution to this convex optimization problem lies at the boundary of Constraint 3, i.e., the solution satisfies all points such that

$$
\beta(1-\delta)=\frac{P_{0}}{P_{t}}
$$

For the solution to lie at the boundary point, we have that $\beta=\frac{P_{0}}{P_{t}(1-\delta)}$. By substituting this expression of $\beta$ in (51), we have that

$$
\int_{0}^{B_{1}} \min \left[\ln \left(1+\frac{\left(\delta P_{t}\right)^{2}}{\sigma_{n_{0}}^{2}}\right), \ln \left(1+\frac{\left(\left(P_{t}(1-\delta)-P_{0}\right) h_{1}\right)^{2}}{\sigma_{n_{1}}^{2}}\right)\right] \Phi_{1} h_{1}^{\theta_{1}^{2} / \sigma_{1}^{2}-1} d h_{1}
$$

As before, the value of $\delta$ that will maximize the integrand is obtained by equating the two arguments of the minimum function:

$$
\ln \left(1+\frac{\left(\delta P_{t}\right)^{2}}{\sigma_{n_{0}}^{2}}\right)=\ln \left(1+\frac{\left(\left(P_{t}(1-\delta)-P_{0}\right) h_{1}\right)^{2}}{\sigma_{n_{1}}^{2}}\right) \Longrightarrow \delta^{*}=\frac{\sigma_{n_{0}} h_{1}\left(P_{t}-P_{0}\right)}{P_{t}\left(\sigma_{n_{1}}+\sigma_{n_{0}} h_{1}\right)}
$$

Here, we note that $\delta^{*}$ is a function of $h_{1}$. However, when $\theta_{1}^{2} \gg \sigma_{1}^{2}, h_{1} \approx B_{1}$, and we have that

$$
\begin{aligned}
\delta^{*} & \approx \frac{\sigma_{n_{0}} B_{1}\left(P_{t}-P_{0}\right)}{P_{t}\left(\sigma_{n_{1}}+\sigma_{n_{0}} B_{1}\right)}, \\
\beta^{*} & \approx \frac{P_{0}}{P_{t}} \frac{1}{\left(1-\delta^{*}\right)}=\frac{P_{0}\left(\sigma_{n_{1}}+\sigma_{n_{0}} B_{1}\right)}{P_{t}\left(\sigma_{n_{1}}+\sigma_{n_{0}} B_{1}\right)-\sigma_{n_{0}} B_{1}\left(P_{t}-P_{0}\right)} .
\end{aligned}
$$


b) Optimization Problem 2:

$$
\begin{array}{ll}
\underset{\delta, \beta}{\operatorname{minimize}} & \operatorname{Pr}(\mathcal{O}) \\
\text { subject to } & \text { i) } \frac{\sqrt{\Upsilon_{\mathrm{th}}} \sigma_{n_{0}}}{P_{t}}<\delta<1, \\
& \text { ii) } 0<\beta<1, \\
& \text { iii) } \beta(1-\delta)>\frac{P_{0}}{P_{t}}
\end{array}
$$

The solution to this optimization problem is straightforward. As can be seen in (54), the outage probability is minimized when both $\delta$ and $\beta$ take on minimum possible values. A minimum value of $\delta: \delta^{*}:=\frac{\sqrt{\Upsilon_{\text {th }}} \sigma_{n_{0}}}{P_{t}}$ will satisfy both Constraint 1 and Constraint 3 of the optimization problem described by (63). However, $\beta$ has to chosen as small as possible but not too small such that Constraint 3 does not hold. Hence, we have that the optimal values of $\delta$ and $\beta$ in this case are given by

$$
\begin{aligned}
& \delta^{*}=\frac{\sqrt{\Upsilon_{\mathrm{th}}} \sigma_{n_{0}}}{P_{t}}, \\
& \beta^{*}=\frac{P_{0}}{P_{t}}\left(\frac{1}{1-\delta^{*}}\right)=\frac{P_{0}}{P_{t}-\sqrt{\Upsilon_{\mathrm{th}}} \sigma_{n_{0}}} .
\end{aligned}
$$

\section{Optimization: $P_{r}<P_{0}$}

Here, we consider the scenario that the total received power $P_{r}<P_{0}$. This constraint occurs due to the fact that the S-R channel is not good enough (for instance there is fog in the channel that can cause a significant loss of signal energy in terms of absorption and scattering) so that received power is not sufficient to ensure that $P_{r} \geq P_{0}$. This implies that the UAV has to land on the ground periodically for charging its battery which leads to a downtime in the link. Therefore, the goal of this section is to maximize the uptime or operational time of the UAV under the constraint that the channel capacity stays above a certain threshold during the uptime of the UAV.

Let us assume that the rotor battery stores $E_{b}$ Joules of energy at full charge which lasts it $T$ seconds of uninterrupted operation if no energy is harvested for rotors. We define $P_{0}:=\frac{E_{b}}{T}$ which is the power or energy consumption rate of the UAV rotor in Watts. Without loss of generality, we further assume that when the UAV is launched in the air for data relaying, its rotor battery is fully charged to $E_{b}$ Joules, whereas the transmitter battery contains zero charge. 
Let $E_{h}:=P_{h} T$ be the proportion of energy harvested during $T$ seconds for powering the rotors. Here, $P_{h}=\beta(1-\delta) P_{r}$, and since $P_{h}<P_{r}<P_{0}$ by assumption, we have that

$$
E_{h}=\beta(1-\delta) P_{r} T<P_{0} T=E_{b}=P_{0} T
$$

Let us define $\Delta_{0}$ as the extra time the UAV can operate after time $T$ due to the harvested energy during interval $[0, T)$. Then

$$
\Delta_{0}=\frac{E_{h}}{P_{0}}=\frac{P_{h} T}{P_{0}}=\left(\frac{P_{h}}{P_{0}}\right) T=\gamma T<T
$$

where

$$
\gamma:=\frac{P_{h}}{P_{0}}=\frac{\beta(1-\delta) P_{r}}{P_{0}}<1
$$

In the same vein, let $\Delta_{1}$ be the extra time the UAV can operate after time $T+\Delta_{0}$ due to energy harvested during interval $\left[T, T+\Delta_{0}\right.$ ), and by a similar argument, we can show that $\Delta_{1}=\gamma \Delta_{0}$, and that $\Delta_{N}=\gamma \Delta_{N-1}$ for any finite positive integer $N$. This means that beginning from time 0 -when the UAV is deployed to close the link — the operational time of the UAV_-before the downtime begins — is

$$
T_{u}=T+\Delta_{0}+\Delta_{1}+\cdots=T+\gamma T+\gamma^{2} T+\gamma^{3} T+\cdots=\frac{T}{1-\gamma}
$$

where $T_{u}$ is the total average time the UAV is able to stay in air in order to relay the data before the downtime begins.

1) Maximization of Uptime $T_{u}$

We want to maximize $T_{u}$ under the minimum ergodic capacity constraint, which implies that we should maximize the harvested power $P_{h}$. Since $P_{r}$ is fixed, we have to maximize the factor $\beta(1-\delta)$ in order to maximize the uptime $T_{u}$. Thus, we have the following optimization problem on our hands:

$$
\begin{array}{ll}
\underset{\delta, \beta}{\operatorname{maximize}} & T_{u} \\
\text { subject to } & i) 0<\delta<1 \\
& \text { ii) } 0<\beta<1, \\
& \text { iii) } \mathcal{C}>\mathcal{C}_{0} .
\end{array}
$$

The goal of this optimization problem is to maximize $\beta$ and minimize $\delta$ for so that the ergodic capacity 
is at least $\mathcal{C}_{0}$. In order to optimize, we note that since it is a convex problem, the solution in terms of the constraint $\mathcal{C}>\mathcal{C}_{0}$ should lie on the boundary, i.e., $\mathcal{C}^{*}=\mathcal{C}_{0}$.

When the approximation $\theta_{1}^{2} \gg \sigma_{1}^{2}$ holds, it is relatively straightforward to find the optimal point. Under this approximation, the ergodic capacity expression in (51) becomes

$$
\mathcal{C} \approx \min \left(\ln \left(1+\frac{\left(\delta P_{t}\right)^{2}}{\sigma_{n_{0}}^{2}}\right), \ln \left(1+\frac{\left((1-\beta)(1-\delta) P_{t} B_{1}\right)^{2}}{\sigma_{n_{1}}^{2}}\right)\right)
$$

and $\mathcal{C}$ is maximized at the point where the two arguments of the minimum function are equal. We note from (71) that increasing the value of $\beta$ will minimize the peak capacity in terms of $\delta$, and that increasing $\beta$ will shift the maximum capacity point towards the left on the $\delta$ axis; that is, towards $\delta$ closer to zero direction (this argument is also supported by the plot in Fig. 5). We note that all the points $(\beta, \delta)$ that intersect the $\mathcal{C}=\mathcal{C}_{0}$ line lie in the boundary $\mathcal{C}^{*}=\mathcal{C}_{0}$. However, there is only one point in the boundary set where the quantity $\beta(1-\delta)$ is maximized, and that point corresponds to the capacity curve whose peak barely touches-or is tangent to-the $\mathcal{C}=\mathcal{C}_{0}$ line.

We know that the ergodic capacity is maximized at the point where the two arguments of the minimum function in (71) are equal. Hence, setting the two arguments equal to each other gives us the first equation in order for us to solve for optimal $\delta$ and $\beta$ :

$$
\frac{\delta}{\sigma_{n_{0}}}=\frac{(1-\beta)(1-\delta) B_{1}}{\sigma_{n_{1}}}
$$

The second equation is obtained by setting the maximum capacity value equal to $\mathcal{C}_{0}$ since the optimal point lies in the boundary $\mathcal{C}=\mathcal{C}_{0}$. This yields us the second equation:

$$
\mathcal{C}_{0}=\ln \left(1+\frac{\left((1-\beta)(1-\delta) P_{t} B_{1}\right)^{2}}{\sigma_{n_{1}}^{2}}\right) \text {. }
$$

We now have two unknowns $(\delta$ and $\beta$ ) and a set of two equations. Solving this simultaneous set of equations leads to the following solution:

$$
\begin{aligned}
\delta^{*} & \approx \frac{\sigma_{n_{0}}}{P_{t}} \sqrt{\exp \left(\mathcal{C}_{0}\right)-1} \\
\beta^{*} & \approx 1-\left(\frac{\sigma_{n_{0}} \sqrt{\exp \left(\mathcal{C}_{0}\right)-1}}{P_{t}-\sigma_{n_{0}} \sqrt{\exp \left(\mathcal{C}_{0}\right)-1}}\right) \frac{\sigma_{n_{1}}}{\sigma_{n_{0}} B_{1}} .
\end{aligned}
$$




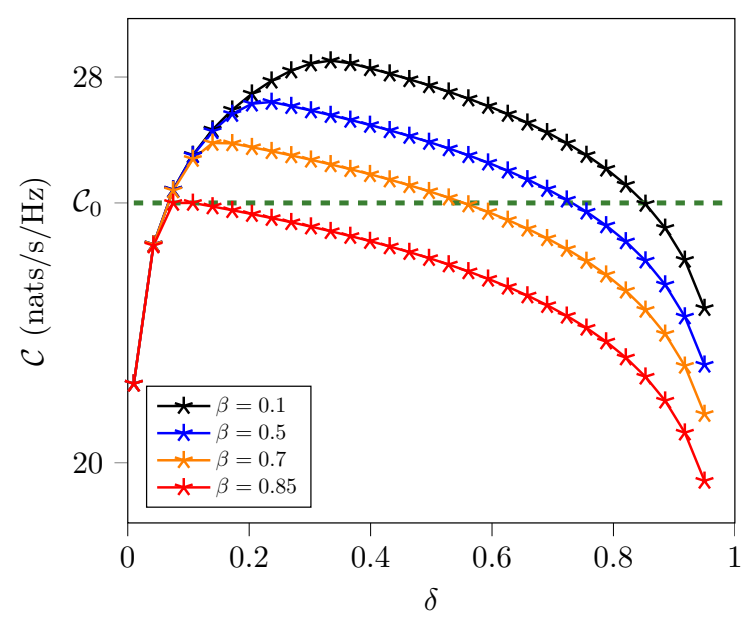

Fig. 5. This figure shows the exact capacity expression as a function of $\delta$ for different values of $\beta$. Other parameter values are as follows: Total distance $D=4000 \mathrm{~m}$, transmitted power $P_{t}=100 \mathrm{~W}$, noise standard deviation at relay is $\sigma_{n_{0}}=2 \times 10^{-5} \mathrm{~V}$, noise standard deviation at the destination receiver is $\sigma_{n_{1}}=1 \times 10^{-6} \mathrm{~V}$, distance $z=2000 \mathrm{~m}$, and the aperture radii $a_{r}=0.2 \mathrm{~m}$ and $a_{d}=1 \mathrm{~m}$.

\section{EXPERIMENTAL RESUlts}

In this section, we will analyze the experimental results of our study in this paper.

\section{A. First Scenario: $P_{0} \approx 0$}

Figure 6 shows the ergodic capacity or maximum achievable rate as a function of the power split factor $\delta$ for different locations of the relay $z$ between the source and the destination. This figure depicts the trend that as the relay is placed closer to the destination, the optimum value of $\delta$ increases (shifts to the right on the $x$ axis). This result can be explained by the following argument: As the distance $z$ increases, the value of factor $B_{1}$ will also increase (please see (9p), which will cause a surge in the value of $\delta$ closer to 1 through Equation (48). In physical terms, what this result says is as follows. As $z$ becomes large, Channel 1 becomes better due to a smaller distance between the relay and destination. An improvement in Channel 1 conditions will lead to a smaller allocation of power to the transmit circuit of the relay since the relay-destination channel is already good, and only a small fraction of power is enough to meet the quality-of-service. This implies that the optimum value of $1-\delta$ will shrink-or the optimum value of $\delta$ will grow-as $z$ becomes large. The same effect can be seen in Figure 7 where a more focused beam in Channel 1 (smaller beamwidth $\theta_{1}$ ) will lead to a larger value for the optimum value of $\delta$.

Figure 8 shows how the optimum value of $\delta$-denoted by $\delta^{*}$-changes with $z$ and beamwidth $\theta_{1}$. A smaller value of $\theta_{1}$ will lead to a higher $\delta^{*}$ and vice versa. In contrast, a larger value of $z$ leads to a larger value of $\delta^{*}$. 


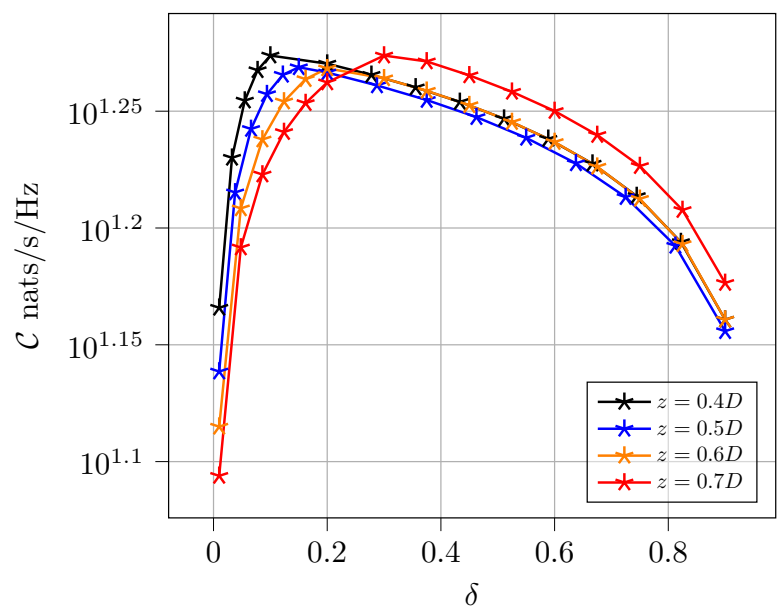

Fig. 6. This figure plots the maximum achievable rate as a function of $\delta$ for different values of $z$. Other parameter values are as follows: Source power $P_{t}=100 \mathrm{~W}$, noise standard deviation $\sigma_{n}=1 \times 10^{-6} \mathrm{~V}$, for Channel 0 , the pointing error standard deviation $\sigma_{0}=1$ mrad and beamwidth $\theta_{0}=2 \mathrm{mrad}$. For Channel 1 , the pointing error standard deviation $\sigma_{1}=0.5 \mathrm{mrad}$, beamwidth $\theta_{1}=0.8 \mathrm{mrad}$ and $\beta=0$.

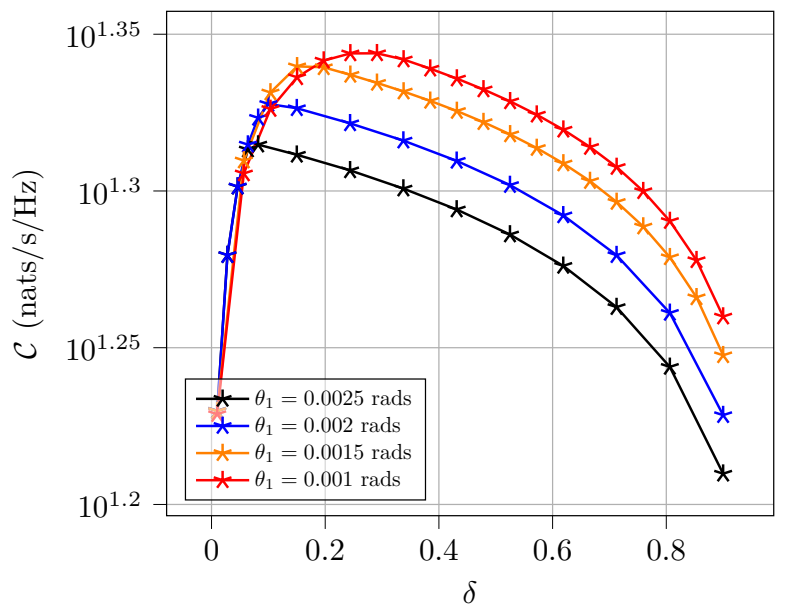

Fig. 7. This figure shows the maximum achievable rate as a function of factor $\delta$ for different values of beamwidth $\theta_{1}$ in Channel 1 . Other parameter values are as follows: Source transmit power $P_{t}=50 \mathrm{~W}$, noise standard deviation $\sigma_{n}=1 \times 10^{-7} \mathrm{~V}$, and for Channel 0 the pointing error standard deviation $\sigma_{0}=1 \mathrm{mrad}$ and beamwidth $\theta_{0}=2 \mathrm{mrad}$. For Channel 1, the pointing error standard deviation $\sigma_{1}=1$ mrad, beamwidth $\theta_{1}=1 \mathrm{mrad}$ and $\beta=0$.

Figure 9 shows the outage probability as a function of $\delta$. We note that a smaller value of angular pointing error standard deviation $\sigma_{1}$ leads to a higher value of optimum $\delta$.

Figure 10 and Figure 11 depict the error probability or the average bit error rate as a function of $\delta$ as a function of source-relay distance $z$ and beamwidth $\theta_{1}$. We note the same trend here as we had discovered for the case of maximum achievable rate: a larger value of $\theta_{1}$ leads to a smaller value of $\delta^{*}$. Conversely, a smaller value of $z$ leads to a smaller value of optimum $\delta$. 


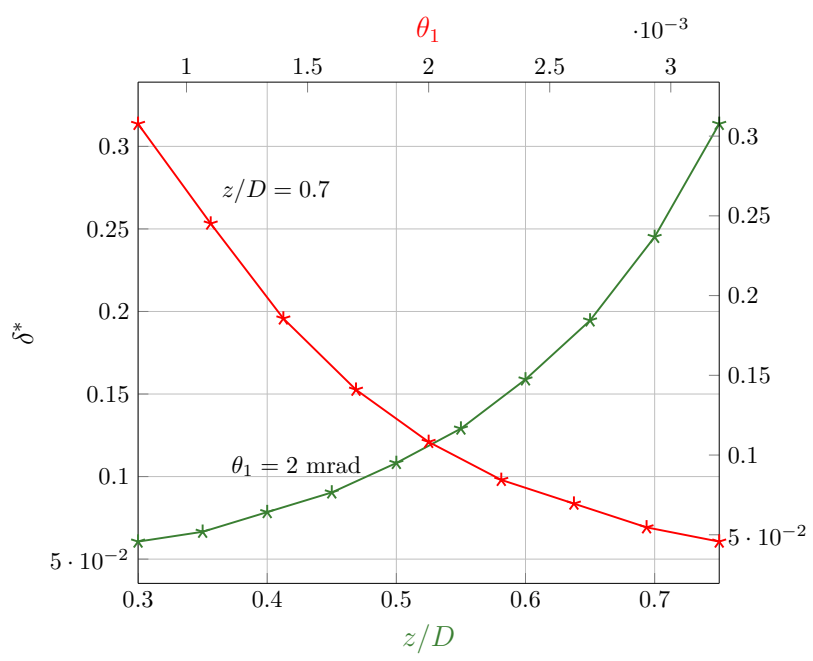

Fig. 8. This figure shows the optimum value of $\delta$ as a function of beamwidth in Channel $1 \theta_{1}$ and the distance ratio $z / D$. Other parameter values are as follows: Source transmit power $P_{t}=50 \mathrm{~W}$, noise standard deviation $\sigma_{n}=1 \times 10^{-7} \mathrm{~V}$, and for Channel 0 the pointing error standard deviation $\sigma_{0}=1 \mathrm{mrad}$ and beamwidth $\theta_{0}=2 \mathrm{mrad}$. For Channel 1, the pointing error standard deviation $\sigma_{1}=1 \mathrm{mrad}$. The value of $\beta=0$.

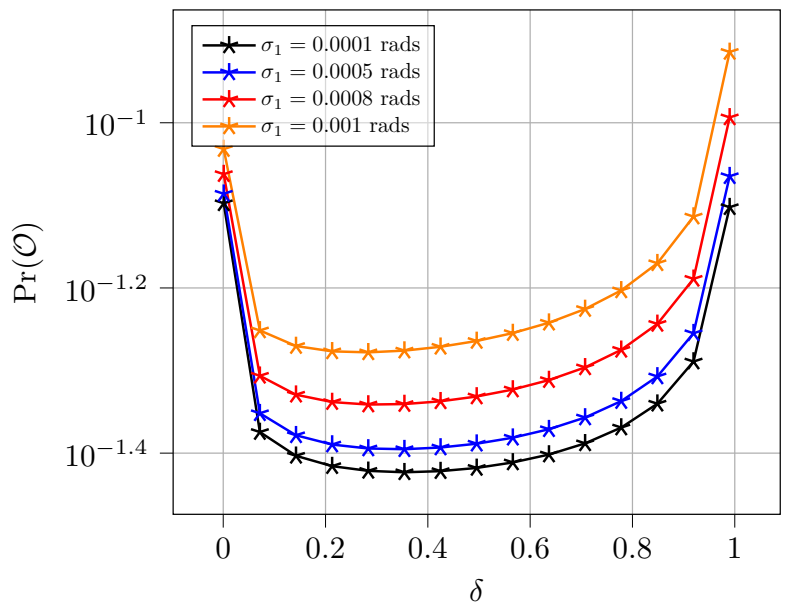

Fig. 9. This figure shows the outage probability as a function of factor $\delta$ for different values of Channel 1 pointing error standard deviation $\sigma_{1}$. Other parameter values are as follows: Total distance $D=4000 \mathrm{~m}$, source transmit power $P_{t}=80 \mathrm{~W}$, noise standard deviation $\sigma_{n}=1 \times 10^{-7} \mathrm{~V}$, Channel 0 parameters are $\theta_{0}=0.5 \mathrm{mrad}$ and $\sigma_{0}=1 \mathrm{mrad}$ and Channel 1 parameters are $\theta_{1}=0.8 \mathrm{mrad}$. The value of threshold $\Upsilon_{\mathrm{th}}=1$ and the distance $z=2800 \mathrm{~m}$.

B. Second Scenario: $0<P_{0}<P_{r}$

In Figure 12, we consider the scenario that the rotor power $P_{0}$ is not negligible and that the total received power by the UAV aperture is large enough so that $P_{r}>P_{0}$. The two subfigures indicate the optimum (maximized) value of maximum achievable rate (left) and the minimum value of outage probability (right) achieved by optimizing over the two dimensional space of $\delta$ and $\beta$. Regarding the maximum achievable rate in Fig. 12, we compare the optimized maximum achievable rates for the exact values of $\delta^{*}$ and $\beta^{*}$ 


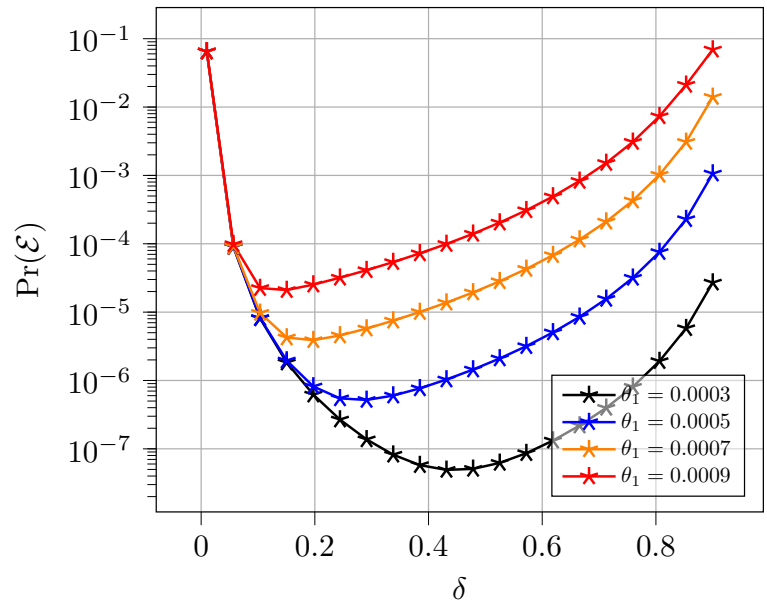

Fig. 10. This figure shows the error probability as a function of factor $\delta$ for different values of beamwidth $\theta_{1}$. Other parameter values are as follows: Total distance $D=5000 \mathrm{~m}$, source transmit power $P_{t}=50 \mathrm{~W}$, noise standard deviation $\sigma_{n}=1 \times 10^{-6} \mathrm{~V}$, channel 0 parameters are $\theta_{0}=8 \mathrm{mrad}$ and $\sigma_{0}=5 \mathrm{mrad}$ and Channel 1 parameters are $\sigma_{1}=0.1 \mathrm{mrad}$. The distance between source and relay is $z=2500 \mathrm{~m}$.

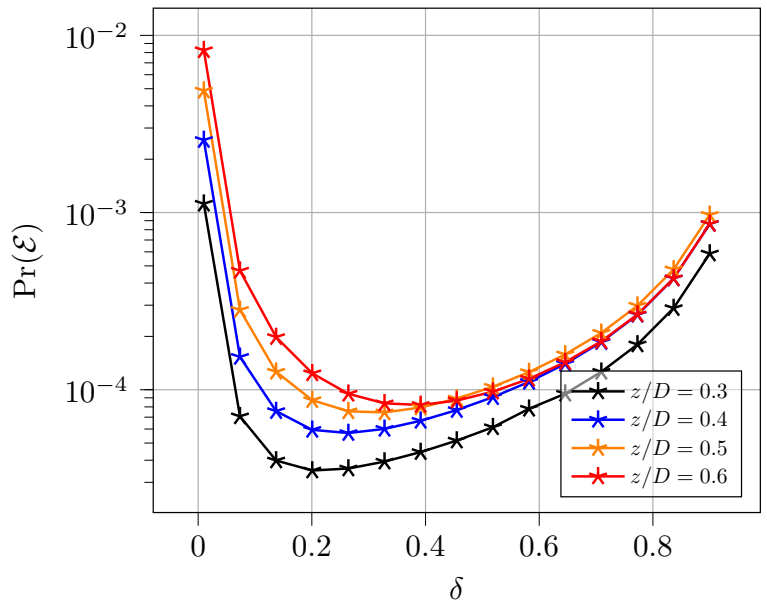

Fig. 11. This figure shows the error probability as a function of factor $\delta$ for different values of source-relay distance $z$. Other parameter values are as follows: Total distance $D=5000 \mathrm{~m}$, source transmit power $P_{t}=50 \mathrm{~W}$, noise standard deviation $\sigma_{n}=1 \times 10^{-6} \mathrm{~V}$, channel 0 parameters are $\theta_{0}=6 \mathrm{mrad}$ and $\sigma_{0}=5 \mathrm{mrad}$ and Channel 1 parameters are $\sigma_{1}=0.1 \mathrm{mrad}$ and $\theta_{1}=0.5 \mathrm{mrad}$.

and the approximate $\left(\delta^{*}, \beta^{*}\right)$ obtained via (61) and (62). As discussed in (V-B3a), the approximation error depends on the ratio $\frac{\theta_{1}^{2}}{\sigma_{1}^{2}}$, and the larger the value of this ratio, the better the approximation. This is corroborated by Figure 12 where the difference between the ergodic capacity curves for the exact and approximate values of $\left(\delta^{*}, \beta^{*}\right)$ shrinks as the ratio $\frac{\theta_{1}^{2}}{\sigma_{1}^{2}}$ gets bigger.

C. Third Scenario: $P_{r}<P_{0}$

Figure 13 shows the maximum achievable uptime that can be obtained when the total received power $P_{r}$ is less than the power $P_{0}$ required to power the rotors of the UAV. These curves plot the total maximum 

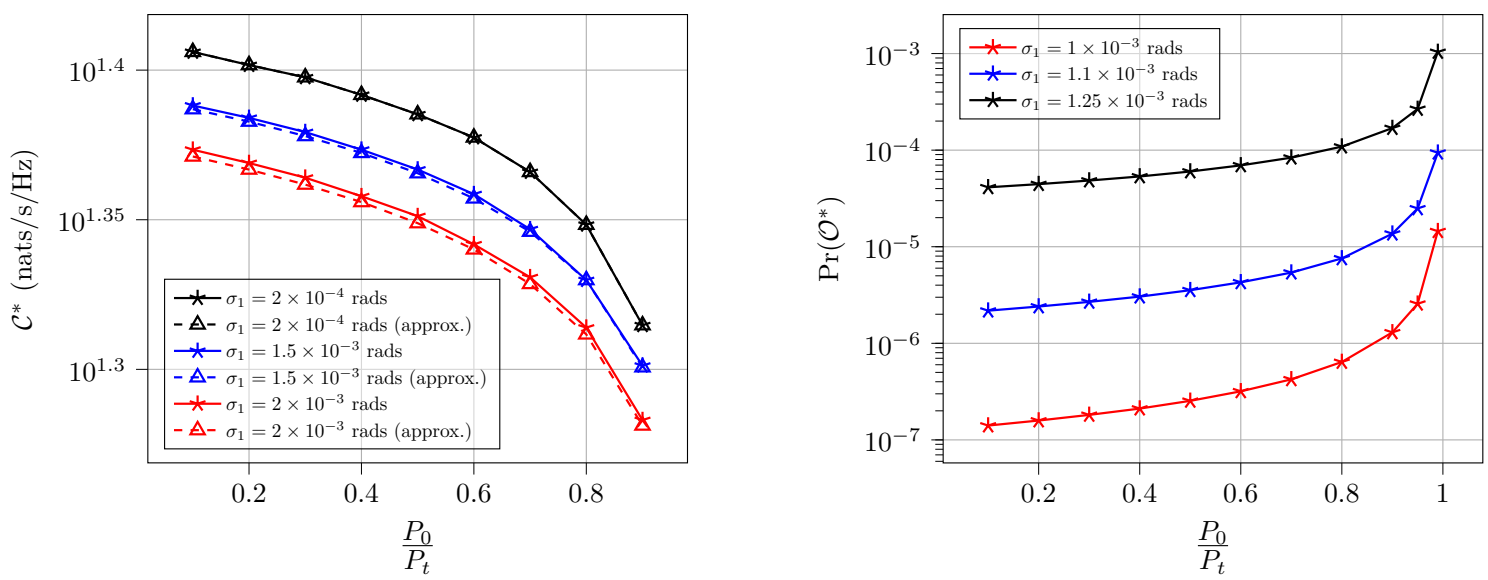

Fig. 12. This figure shows the optimum value of maximum achievable rate (left) and the minimum value of outage probability (right) as a function of power ratio $P_{0} / P_{t}$. In these plots, the maximum achievable rate and the outage probability are optimized over the two dimensional space $(\delta, \beta)$. Other parameter values are as follows: Total link distance $D=4000 \mathrm{~m}$, source transmit power $P_{t}=100 \mathrm{~W}$, noise standard deviation in Channel 0 is $\sigma_{n_{0}}=100 \mu \mathrm{V}$, noise standard deviation in Channel 1 is $\sigma_{n_{1}}=5 \mu \mathrm{V}$, Channel 1 parameters are $\sigma_{1}=1.5$ mrad and $\theta_{1}=2 \mathrm{mrad}$ and source-relay distance $z=2000 \mathrm{~m}$.

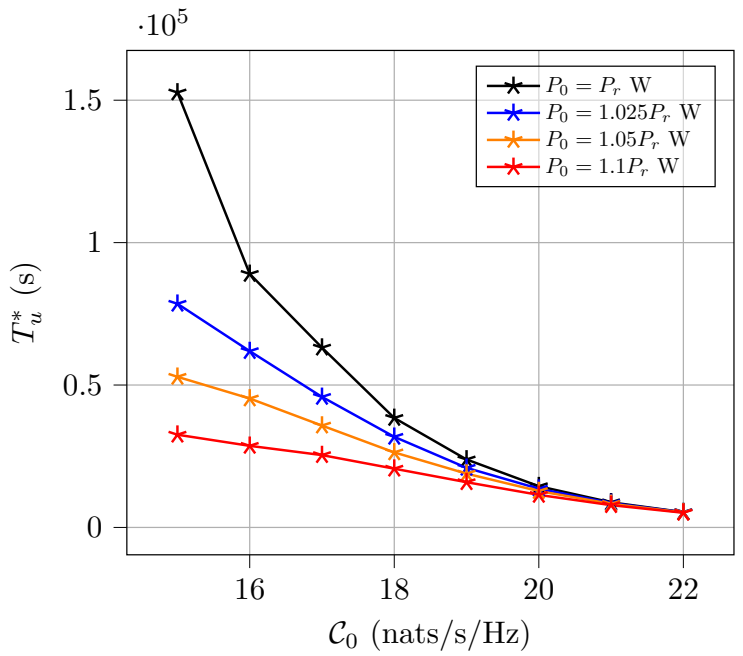

Fig. 13. This figure shows the maximized value of uptime $T_{u}^{*}$ for different values of power $P_{0}$ under the constraint that $\mathcal{C} \geq \mathcal{C}_{0}$. Other parameter values are as follows: Total distance $D=5000 \mathrm{~m}$, source transmit power $P_{t}=100 \mathrm{~W}$, noise standard deviation in Channel 0 $\sigma_{n_{0}}=1 \mathrm{mV}$ and noise standard deviation in Channel $1 \sigma_{n_{1}}=1 \mu \mathrm{V}$. The Channel 1 parameters are $\sigma_{1}=1 \mathrm{mrad}$ and $\theta_{1}=2 \mathrm{mrad}$. The value of $z=2500 \mathrm{~m}$ and the time parameter $T=3600 \mathrm{~s}$.

achievable uptime as a function of capacity constraint $\mathcal{C}_{0}$. With an increase in the value of $P_{0}$, there is a subsequent decrease in the total uptime of the UAV.

\section{CONCLUSION}

In this study, we have analyzed the energy optimization problem for a laser-powered UAV decodeand-forward relay. The goal of this study was to optimally divide or split the beam energy between the 
decoding, transmission and rotor assemblies of the relay while maximizing one of the following qualityof-service metrics: the maximum achievable rate, the outage probability and the error probability. We discussed three important optimization scenarios: when the UAV rotor power was negligible, the scenario where the received energy was larger than the required rotor power, and finally the case when the required rotor power was larger than the received power. We were able to obtain closed-form expressions for $\delta^{*}$ and $\beta^{*}$ for all the aforementioned scenarios under the assumption that the ratio $\frac{\theta_{1}^{2}}{\sigma_{1}^{2}}$ was larger than unity.

In the future study, we want to work on the energy optimization problem for UAV relays while taking into consideration the composite channel that accounts for both the pointing error and the scintillation phenomenon due to atmosphere. We are particularly interested in modeling scintillation with Exponentiated Weibull fading model which has been shown to model the weak to strong atmospheric turbulence very effectively [33]. Additionally, a more general model of the pointing error, such as the one discussed in [34], will be used instead of the Rayleigh model for the purpose of energy optimization. Finally, since the optimization scheme is heavily dependent on the received signal parameters - such as the pointing error statistics in both S-R and R-D channels-the future study will also propose low complexity estimation schemes that will be used to estimate the channel parameters in real-time.

\section{REFERENCES}

[1] Y. Zeng, R. Zhang, and T. J. Lim, "Wireless communications with unmanned aerial vehicles: opportunities and challenges," IEEE Communications Magazine, vol. 54, pp. 36-42, 2016.

[2] W. Saad, M. Bennis, M. Mozaffari, and X. Lin, Wireless Communications and Networking for Unmanned Aerial Vehicles. Cambridge University Press, 2020.

[3] M. T. Dabiri, S. M. S. Sadough, and M. A. Khalighi, "Channel modeling and parameter optimization for hovering uav-based free-space optical links,” IEEE Journal on Selected Areas in Communications, vol. 36, no. 9, pp. 2104-2113, 2018.

[4] M. A. Kishk, A. Bader, and M.-S. Alouini, "On the 3-D placement of airborne base stations using tethered uavs," IEEE Transactions on Communications, vol. 68, no. 8, pp. 5202-5215, 2020.

[5] I. Krikidis, S. Timotheou, S. Nikolaou, G. Zheng, D. W. K. Ng, and R. Schober, "Simultaneous wireless information and power transfer in modern communication systems," IEEE Communications Magazine, vol. 52, no. 11, pp. 104-110, 2014.

[6] P. D. Diamantoulakis, K. N. Pappi, Z. Ma, X. Lei, P. C. Sofotasios, and G. K. Karagiannidis, "Airborne radio access networks with simultaneous lightwave information and power transfer (slipt)," in 2018 IEEE Global Communications Conference (GLOBECOM), 2018, pp. 1-6.

[7] P. D. Diamantoulakis and G. K. Karagiannidis, "Simultaneous lightwave information and power transfer (slipt) for indoor iot applications," in GLOBECOM 2017 - 2017 IEEE Global Communications Conference, 2017, pp. 1-6.

[8] G. Pan, P. D. Diamantoulakis, Z. Ma, Z. Ding, and G. K. Karagiannidis, "Simultaneous lightwave information and power transfer: Policies, techniques, and future directions," IEEE Access, vol. 7, pp. 28 250-28 257, 2019. 
[9] S. Ma, F. Zhang, H. Li, F. Zhou, Y. Wang, and S. Li, "Simultaneous lightwave information and power transfer in visible light communication systems," IEEE Transactions on Wireless Communications, vol. 18, no. 12, pp. 5818-5830, 2019.

[10] A. M. Abdelhady, O. Amin, B. Shihada, and M.-S. Alouini, "Spectral efficiency and energy harvesting in multi-cell slipt systems," IEEE Transactions on Wireless Communications, vol. 19, no. 5, pp. 3304-3318, 2020.

[11] F. Benkhelifa, A. K. S. Salem, and M.-S. Alouini, "Simultaneous wireless information and power transfer for decode-and-forward mimo relay communication systems," in 2015 IEEE 81st Vehicular Technology Conference (VTC Spring), 2015, pp. 1-5.

[12] D. K. P. Asiedu, H. Lee, and K.-J. Lee, "Simultaneous wireless information and power transfer for decode-and-forward multihop relay systems in energy-constrained iot networks," IEEE Internet of Things Journal, vol. 6, no. 6, pp. 9413-9426, 2019.

[13] C. Peng, G. Wang, F. Li, and H. Liu, "Joint resource allocation for swipt-based two-way relay networks," Energies, vol. 13, no. 22, 2020. [Online]. Available: https://www.mdpi.com/1996-1073/13/22/6024

[14] M.-A. Lahmeri, M. A. Kishk, and M.-S. Alouini, "Stochastic geometry-based analysis of airborne base stations with laser-powered uavs," IEEE Communications Letters, vol. 24, pp. 173-177, 2020.

[15] M. S. Bashir and M.-S. Alouini, "Optimal placement of uav-assisted amplify-and-forward relays for mitigation of pointing error in optical wireless backhaul," Sep 2021. [Online]. Available: https://www.techrxiv.org/articles/preprint/Optimal_Placement_of_ UAV-Assisted_Amplify-and-Forward_Relays_for_Mitigation_of_Pointing_Error_in_Optical_Wireless_Backhaul/16550916/1

[16] E. Zedini and M.-S. Alouini, "Multihop relaying over im/dd fso systems with pointing errors," J. Lightw. Technol., vol. 33, no. 23, pp. $5007-5015,2015$.

[17] E. Zedini, H. Soury, and M.-S. Alouini, "On the performance analysis of dual-hop mixed FSO/RF systems," IEEE Trans. Wireless Commun., vol. 15, no. 5, pp. 3679-3689, 2016.

[18] M. S. Bashir and M. R. Bell, "Optical beam position estimation in free-space optical communication," IEEE Transactions on Aerospace and Electronic Systems, vol. 52, no. 6, December 2016.

[19] _ - "Optical beam position tracking in free-space optical communication systems," IEEE Transactions on Aerospace and Electronic Systems, vol. 20, no. 2, April 2018.

[20] M. S. Bashir and M. R. Bell, "The impact of optical beam position estimation on the probability of error in free-space optical communications," IEEE Transactions on Aerospace and Electronic Systems, vol. 55, no. 3, pp. 1319-1333, June 2019.

[21] M. S. Bashir and S. S. Muhammad, "Time synchronization in photon-limited deep space optical communications," IEEE Transactions on Aerospace and Electronic Systems, vol. 56, no. 1, pp. 30-40, 2020.

[22] M. S. Bashir, "Free-space optical communications with detector arrays: A mathematical analysis," IEEE Transactions on Aerospace and Electronic Systems, vol. 56, no. 2, pp. 1420-1429, 2020.

[23] M. S. Bashir and M.-S. Alouini, "Signal acquisition with photon-counting detector arrays in free-space optical communications," IEEE Transactions on Wireless Communications, vol. 19, no. 4, pp. 2181-2195, 2020.

[24] M. S. Bashir and M. -S. Alouini, "Adaptive acquisition schemes for photon-limited free-space optical communications," IEEE Transactions on Communications, January 2020, submitted for publication (available on arXiv at http://arxiv.org/abs/2001.04010).

[25] M. S. Bashir and M. S. Alouini, "Free-space optical MISO communications with an array of detectors," IEEE Open Journal of the Communications Society, vol. 1, pp. 1765-1780, 2020.

[26] R. Boluda-Ruiz, A. García-Zambrana, C. Castillo-Vázquez, B. Castillo-Vázquez, and S. Hranilovic, "Outage performance of exponentiated weibull fso links under generalized pointing errors," Journal of Lightwave Technology, vol. 35, no. 9, pp. 1605-1613, 2017.

[27] A. A. Farid and S. Hranilovic, "Outage capacity optimization for free-space optical links with pointing errors," Journal of Lightwave Technology, vol. 25, no. 7, July 2007. 
[28] Z. Wang, D. Tsonev, S. Videv, and H. Haas, "On the design of a solar-panel receiver for optical wireless communications with simultaneous energy harvesting," IEEE Journal on Selected Areas in Communications, vol. 33, no. 8, pp. 1612-1623, 2015.

[29] M. S. Bashir and M.-S. Alouini, "Optimal power allocation between beam tracking and symbol detection channels in a free-space optical communications receiver," IEEE Transactions on Communications, pp. 1-1, 2021.

[30] A. Thibbotuwawa, P. Nielsen, B. Zbigniew, and G. Bocewicz, "Energy consumption in unmanned aerial vehicles: A review of energy consumption models and their relation to the uav routing," in Information Systems Architecture and Technology: Proceedings of 39th International Conference on Information Systems Architecture and Technology - ISAT 2018, J. Świątek, L. Borzemski, and Z. Wilimowska, Eds. Cham: Springer International Publishing, 2019, pp. 173-184.

[31] S. Das, E. Poves, J. Fakidis, A. Sparks, S. Videv, and H. Haas, "Towards energy neutral wireless communications: Photovoltaic cells to connect remote areas," Energies, vol. 12, no. 19, 2019. [Online]. Available: https://www.mdpi.com/1996-1073/12/19/3772

[32] S. Das, A. Sparks, E. Poves, S. Videv, J. Fakidis, and H. Haas, "Effect of sunlight on photovoltaics as optical wireless communication receivers," Journal of Lightwave Technology, vol. 39, no. 19, pp. 6182-6190, 2021.

[33] R. Barrios and F. Dios, "Exponentiated weibull distribution family under aperture averaging for gaussian beam waves," Opt. Express, vol. 20, no. 12, pp. 13 055-13 064, Jun 2012. [Online]. Available: http://www.opticsexpress.org/abstract.cfm?URI=oe-20-12-13055

[34] C. B. Issaid, K.-H. Park, and M.-S. Alouini, "A generic simulation approach for the fast and accurate estimation of the outage probability of single hop and multihop FSO links subject to generalized pointing errors," IEEE Trans. Wireless Commun., vol. 16, no. 10, pp. 6822-6837, 2017. 